\title{
Chloroplasts alter their morphology and accumulate at the pathogen interface during infection by Phytophthora infestans
}

\author{
Zachary Savage $^{1, \dagger}$ (D), Cian Duggan ${ }^{1,+}$, Alexia Toufexi $^{1}$, Pooja Pandey ${ }^{1}$, Yuxi Liang ${ }^{1}$, María Eugenia Segretin ${ }^{3}$, \\ Lok Him Yuen ${ }^{1}$, David C. A. Gaboriau ${ }^{2}$, Alexandre Y. Leary ${ }^{1}$, Yasin Tumtas ${ }^{1}$, Virendrasinh Khandare ${ }^{1}$, Andrew D. Ward ${ }^{4}$, \\ Stanley W. Botchway ${ }^{4}$, Benji C. Bateman ${ }^{4}$, Indranil Pan ${ }^{5,6}$, Martin Schattat ${ }^{7}$, Imogen Sparkes ${ }^{8}$ and Tolga O. Bozkurt ${ }^{1, *}$ \\ ${ }^{1}$ Department of Life Sciences, Imperial College London, Imperial College Road, South Kensington Campus, London, London \\ SW7 2AZ, UK, \\ ${ }^{2}$ Facility for Imaging by Light Microscopy, Faculty of Medicine, National Heart \& Lung Institute (NHLI), Imperial College \\ London, South Kensington, SAF building, London SW7 2AZ, UK, \\ ${ }^{3}$ Instituto de Investigaciones en Ingeniería Genética y Biología Molecular 'Dr Héctor N. Torres' (INGEBI)-Consejo Nacional \\ de Investigaciones Científicas y Técnicas (CONICET), Vuelta de Obligado 2490, Ciudad Autónoma de Buenos Aires \\ C1428ADN, Argentina, \\ ${ }^{4}$ Central Laser Facility, Science and Technology Facilities Council Harwell, Science and Technology Facilities Council, Ruther- \\ ford Appleton Laboratory, Harwell, Didcot OX11 0QX, UK, \\ ${ }^{5}$ Centre for Process Systems Engineering and Centre for Environmental Policy, Imperial College London, South Kensington \\ Campus, London, London SW7 2AZ, UK, \\ ${ }^{6}$ The Alan Turing Institute, British Library, 96 Euston Road, London, London NW1 2DB, UK, \\ ${ }^{7}$ Martin Luther Universität Halle-Wittenberg, Halle 06108 Halle, Germany, and \\ ${ }^{8}$ School of Biological Sciences, University of Bristol, University of Bristol, St Michael's Hill, Bristol BS8 8DZ, UK
}

Received 29 March 2021; revised 2 July 2021; accepted 8 July 2021; published online 12 July 2021.

*For correspondence (e-mail o.bozkurt@imperial.ac.uk).

${ }^{\dagger}$ These authors contributed equally to this work.

\section{SUMMARY}

Upon immune activation, chloroplasts switch off photosynthesis, produce antimicrobial compounds and associate with the nucleus through tubular extensions called stromules. Although it is well established that chloroplasts alter their position in response to light, little is known about the dynamics of chloroplast movement in response to pathogen attack. Here, we report that during infection with the Irish potato famine pathogen Phytophthora infestans, chloroplasts accumulate at the pathogen interface, associating with the specialized membrane that engulfs the pathogen haustorium. The chemical inhibition of actin polymerization reduces the accumulation of chloroplasts at pathogen haustoria, suggesting that this process is partially dependent on the actin cytoskeleton. However, chloroplast accumulation at haustoria does not necessarily rely on movement of the nucleus to this interface and is not affected by light conditions. Stromules are typically induced during infection, embracing haustoria and facilitating chloroplast interactions, to form dynamic organelle clusters. We found that infection-triggered stromule formation relies on BRASSINOSTEROID INSENSITIVE 1-ASSOCIATED KINASE 1 (BAK1)-mediated surface immune signaling, whereas chloroplast repositioning towards haustoria does not. Consistent with the defense-related induction of stromules, effector-mediated suppression of BAK1-mediated immune signaling reduced stromule formation during infection. On the other hand, immune recognition of the same effector stimulated stromules, presumably via a different pathway. These findings implicate chloroplasts in a polarized response upon pathogen attack and point to more complex functions of these organelles in plant-pathogen interactions.

Keywords: haustorium, stromule, Phytophthora infestans, chloroplast movement, laser capture, focal immunity, effectors. 


\section{INTRODUCTION}

Phytophthora infestans is an oomycete pathogen that causes potato late blight, one of the most historically important and economically devastating crop diseases. The pathogen penetrates host cells via haustoria, which are infection structures that extend from its intercellular invasive hyphae. Haustoria are surrounded by the plantderived extrahaustorial membrane (EHM), across which effectors secreted by the pathogen translocate inside the host cell (Wang et al., 2017; Whisson et al., 2007, 2016). This interface is key to the success or failure of infection and is therefore targeted by focal immune responses of the plant (Bozkurt et al., 2011; Dagdas et al., 2018; Kwon et al., 2008). This includes the deposition of callose, the redirection of autophagy and movement of the nucleus towards the site of penetration (Dagdas et al., 2018; Griffis et al., 2014; Wang et al., 2021). Although continuous with the plasma membrane, there is a stark difference between the biochemical composition of the EHM and that of the plasma membrane (Bozkurt et al., 2014, 2015).

The EHM typically lacks the surface localized pattern recognition receptors (PRRs), which activate downstream immune responses through the recognition of pathogenassociated molecular patterns (PAMPs) (Bozkurt et al., 2014, 2015). Once PPRs detect PAMPs, downstream signaling is triggered, often in coordination with a co-receptor such as BRASSINOSTEROID INSENSITIVE 1-ASSOCIATED RECEPTOR KINASE 1 (BAK1), to induce basal immune responses (Chaparro-Garcia et al., 2011; Heese et al., 2007). Basal responses are those that make up the first line of defense against a range of pathogens (specialist and generalist) following cell surface immune activation from the detection of PAMPs. To counteract this, pathogens typically deploy host-translocated effectors to subvert surfacemediated immunity. For example, $P$. infestans hosttranslocated RXLR effector AVR3a suppresses the BAK1mediated surface immune response (Chaparro-Garcia et al., 2015). However, presumably, plant basal responses still contribute to immunity against adapted pathogens, as the immune suppression by effectors is often partial, as in the case of AVR3a (Chaparro-Garcia et al., 2015). Moreover, the downregulation of $B A K 1$ gene expression in the solanaceous model plant Nicotiana benthamiana leads to significantly enhanced pathogen growth, further highlighting the importance of surface-mediated immunity against P. infestans (Chaparro-Garcia et al., 2015). This makes $N$. benthamiana an excellent model for studies to dissect the functional principles of basal plant immunity against $P$. infestans. Furthermore, $N$. benthamiana lacks the specialized nucleotide-binding leucine-rich repeat (NLR) type of immune receptors that can sense $P$. infestans effectors intracellularly. This is also advantageous because it allows for live cell imaging of $P$. infestans infection, as NLR- mediated immunity often triggers a form of programed cell death at the site of infection, known as the hypersensitive response (HR) (Wu et al., 2017).

The activation of immunity at the cell surface stimulates chloroplasts to shut down photosynthesis, synthesize defense hormone precursors and generate reactive oxygen species (ROS) (Padmanabhan and Dinesh-Kumar, 2010; Su et al., 2018), indicating that chloroplasts are major components of the plant defense system. Pathogens are known to target chloroplasts with effector proteins, further highlighting their importance in immunity (Jelenska et al., 2007; Pecrix et al., 2019; Petre et al., 2016; Zabala et al., 2015). Interestingly, several genes associated with resistance to oomycete pathogens were found to encode chloroplastlocalized proteins (Belhaj et al., 2009; Van Damme et al., 2009). Chloroplasts also produce stroma-filled tubules (stromules) in response to a range of elicitors, including phytohormones, ROS and the bacterial PAMP flg22 (Brunkard et al., 2015; Caplan et al., 2015; Gray et al., 2012). Although the exact function(s) of stromules is still unclear, they have been implicated in immunity, chloroplast movement and connection to the plant cell nucleus (Caplan et al., 2015; Kumar et al., 2018). Immune stimulation by PAMPs and ROS also induce the association between chloroplasts and the nucleus, hinting at potential defenserelated roles of chloroplast redistribution during infection (Ding et al., 2019). However, the molecular and physiological mechanism of how chloroplast immunity is launched against invading pathogens is unclear.

Here, we used quantitative confocal microscopy to investigate the spatial dynamics of chloroplasts in living plant cells infected by $P$. infestans. We show that chloroplasts accumulate around haustoria in a dynamic fashion, but that this process does not necessarily rely on the movement of the nucleus towards the haustorium. We found that the actin cytoskeleton, but not light conditions, are critical for chloroplast positioning around the haustorium. Our microscopy analyses using optical tweezers suggest an association between chloroplasts and the EHM. Finally, we demonstrate that chloroplasts also alter their morphology by the induction of stromules as a defense response, whereas effectors can counteract this process.

\section{RESULTS}

\section{Chloroplasts accumulate at the host-pathogen interface in an actin-dependent manner, but irrespective of light conditions}

Whereas the immune-related roles of chloroplasts in producing antimicrobial compounds and defense signaling molecules are well established, little is known on the subcellular dynamics of chloroplast movement and stromule induction during infection. To gain insights into this process, we monitored the live infection of $N$. benthamiana by 
$P$. infestans, specifically targeting cells containing a pathogen haustorium (haustoriated cells). In this infection model, haustoria are generally visible in the host epidermal cells, where the chloroplasts are smaller and less abundant than in the mesophyll. We reasoned that the more sparsely distributed chloroplasts of these epidermal cells may redistribute towards the site of infection to partake in a localized intracellular immune response.

During the infection of $N$. benthamiana with the fluorescently tagged strain of $P$. infestans, 88069td, haustoria are easily visible in host cells. Confocal microscopy of infected leaf epidermal cells stably expressing GFP in chloroplast stroma (CpGFP herein) revealed that chloroplasts associate with $40 \%$ of haustoria ( $n=280$ haustoria) (Figure 1a; Video Clip S1). Haustoria were often associated with multiple chloroplasts, and these chloroplasts were often mobile around the site of infection (Figure 1a; Video Clip S2).

We investigated whether the positioning of chloroplasts around haustoria was a response to infection or the result of chance encounter, i.e. through haustoria coincidentally penetrating cells at positions where there are chloroplasts. To check whether this association occurred at a greater frequency than would be expected by random chance encounter, we developed an unbiased method to position 'mock' haustoria throughout micrographs of infected tissue. For each real haustorium identified in an image, a straight line was drawn, beginning at the point that the haustorium entered the cell and ending at the cell periphery, opposite the haustorium entry point; this end point was imagined as the position of a mock haustorial penetration. We then categorized whether each mock haustorium was immediately adjacent to, or in contact with, a chloroplast in that position (for a visual representation of the approach, see Figure S1a). If chloroplast accumulation at haustoria was random, we would expect rates of actual and mock haustoria to be the same on opposite sides of the cell. Compared with the actual haustoria, there were significantly fewer instances of contact between chloroplasts and mock haustoria (Figure S1b), suggesting the association between haustoria and chloroplasts was not through random encounters during cell penetration.

To gain further insights into chloroplast positioning around haustoria, we monitored chloroplast dynamics in haustoriated cells with time-lapse microscopy. Here, we observed several instances of chloroplast movement towards haustoria, suggesting an active relocation of chloroplasts towards the site of intracellular infection (Video Clips S3 and S4). Similar observations were made during wild-type $P$. infestans infection, visualizing the EHM with REMORIN 1.3 (RFP:REM1.3) (Video Clip S5). Additionally, we observed some instances where haustoria that were associated with chloroplasts collapse during the acquisition of the time lapse (Video Clips S6 and S7). This indicates that some of the haustoria-chloroplast associations could be missed out during image quantification, as it is not always possible to identify collapsed haustoria. The chance observation of a haustorium collapsing in real time was rare, and we therefore did not attempt to assess quantitatively whether chloroplast-haustoria association correlated with the events of collapse.

The cytoskeleton, and particularly actin, has known roles in chloroplast movement (Wada and Kong, 2018), nucleus movement (Higa et al., 2014), stromule interactions (Erickson et al., 2018; Kumar et al., 2018) and cell polarization towards the sites of pathogen penetration (Kobayashi and Hakuno, 2003; Opalski et al., 2005). Therefore, we next tested whether the chemical inhibition of actin polymerization by latrunculin A (LatA) could influence the accumulation of chloroplasts at haustoria. To monitor the successful disruption of actin, a GFP-tagged actin chromobody was transiently expressed during treatment with $1 \mu \mathrm{M}$ LatA of infected wild-type plants by $P$. infestans 88069td (Figure $1 b)$. We also tested a range of LatA concentrations for the ability to visually disrupt actin filaments before completing the infection microscopy, in order to use a minimal concentration of LatA (Figure S2). Treatment with LatA significantly reduced the frequency of chloroplast-haustoria associations compared with control conditions $134 \%$ of $n=148$ haustoria and $47 \%$ of $n=93$ haustoria, respectively) (Figure 1c). This points to a role for actin in the accumulation of chloroplasts at haustoria. However, we are cautious of overinterpreting these data because of the non-specific nature of the drug and its plausible ability to influence the growth and virulence of the pathogen (Ketelaar et al., 2012). By using the GFP marker to ensure the inhibition of actin in the cells being imaged, we sacrificed the ability to monitor stromule induction during this experiment. We also tested the effect of treatment with the chemical microtubule disruptor oryzalin $(100 \mu \mathrm{M})$ after $24 \mathrm{~h}$, but found no significant difference in chloroplasthaustoria association between treatment and control conditions (Figure S3a). However, treatment with $100 \mu \mathrm{M}$ of oryzalin appeared to be ineffective at $24 \mathrm{~h}$ as no disruption of the microtubule marker TUA5-GFP was observed after $24 \mathrm{~h}$ (Figure S3b), despite observing the clear disruption of microtubules at $3 \mathrm{~h}$ (Figure S3d,e), as previously validated (Erickson et al., 2018). We repeated the stromule induction experiments after $3 \mathrm{~h}$ of treatment with $100 \mu \mathrm{M}$ oryzalin and found no effect (Figure S3f,g). We did not conduct the chloroplast-haustoria association experiment after $3 \mathrm{~h}$ of treatment as we reasoned that this time period was likely to be too short to allow noticeable disruption in chloroplast positioning.

Finally, we also tested whether light exposure has any impact on the accumulation of chloroplasts at haustoria. For dark conditions, we kept leaves of CpGFP plants infected with $P$. infestans 88069td in the dark for 2 days prior to imaging (dark-dark), whereas we kept the control 
(a) $40 \%$ of $\mathbf{N}=\mathbf{2 8 0}$ haustoria associated with chloroplast
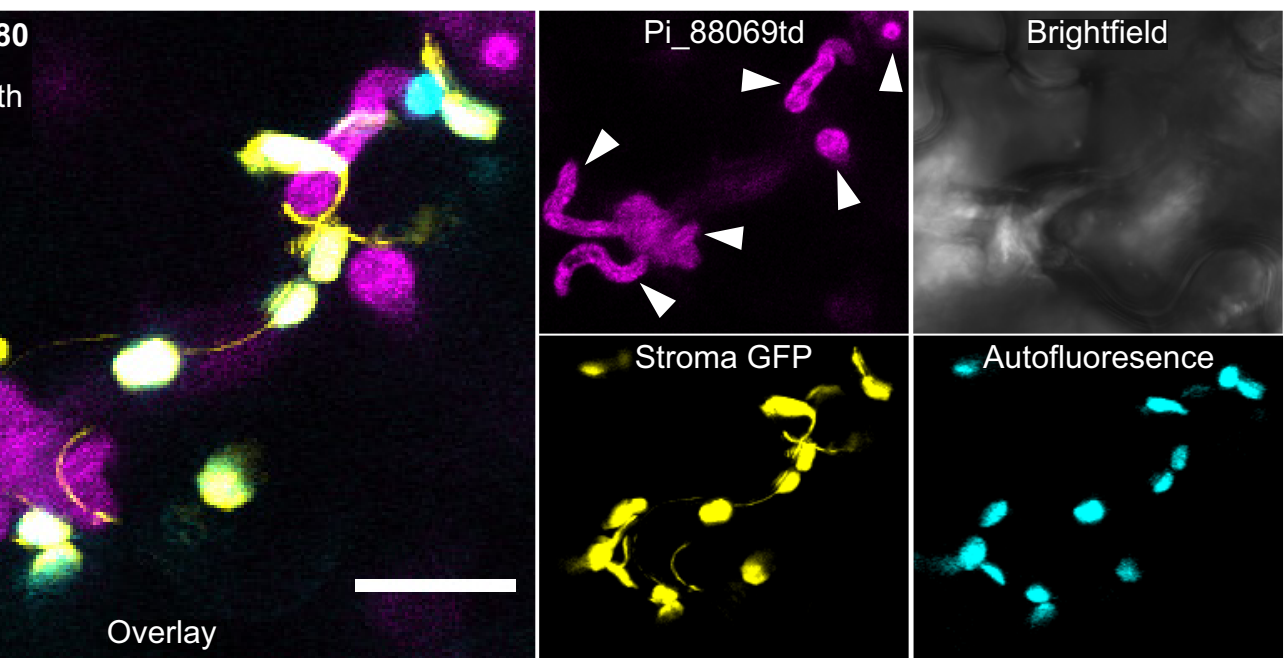

(b)
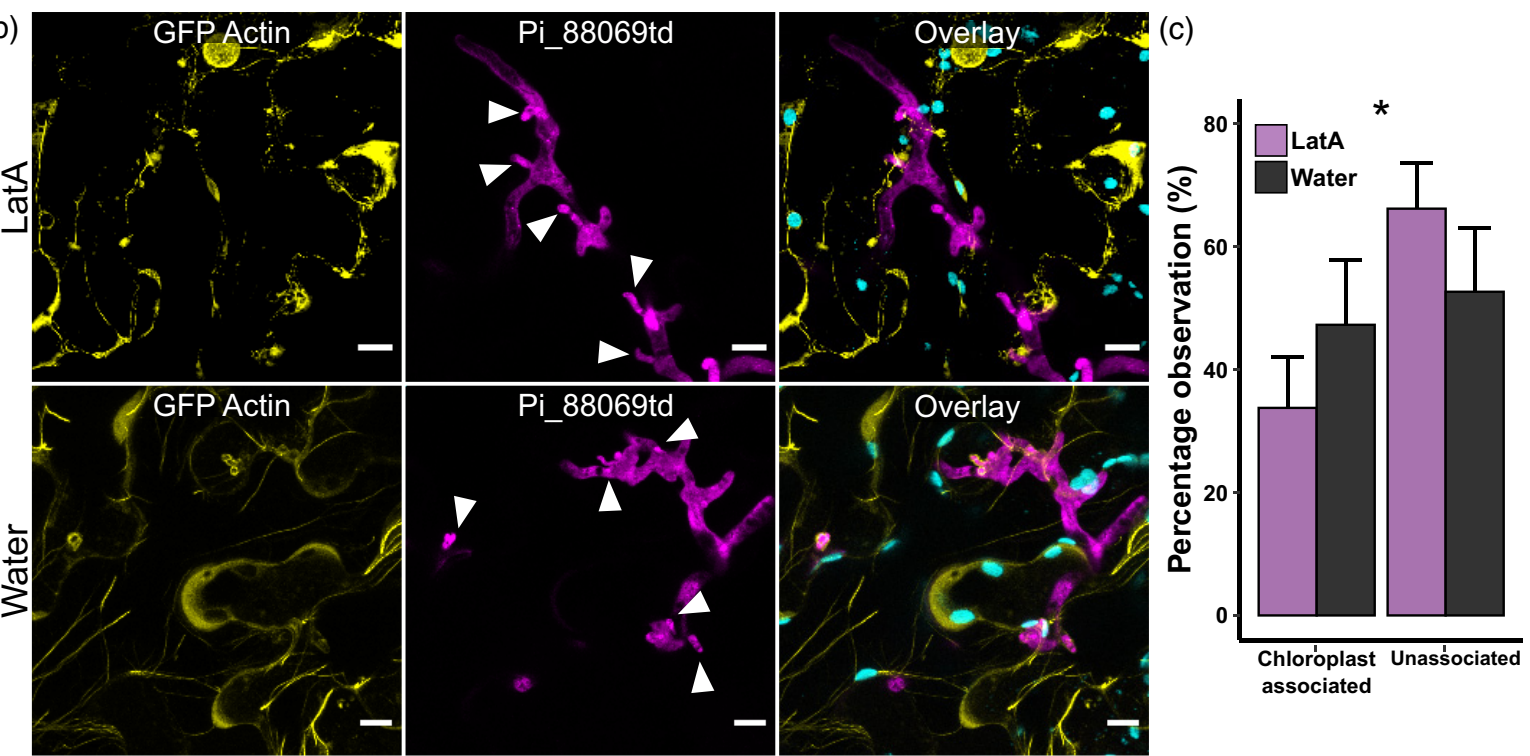

(d)
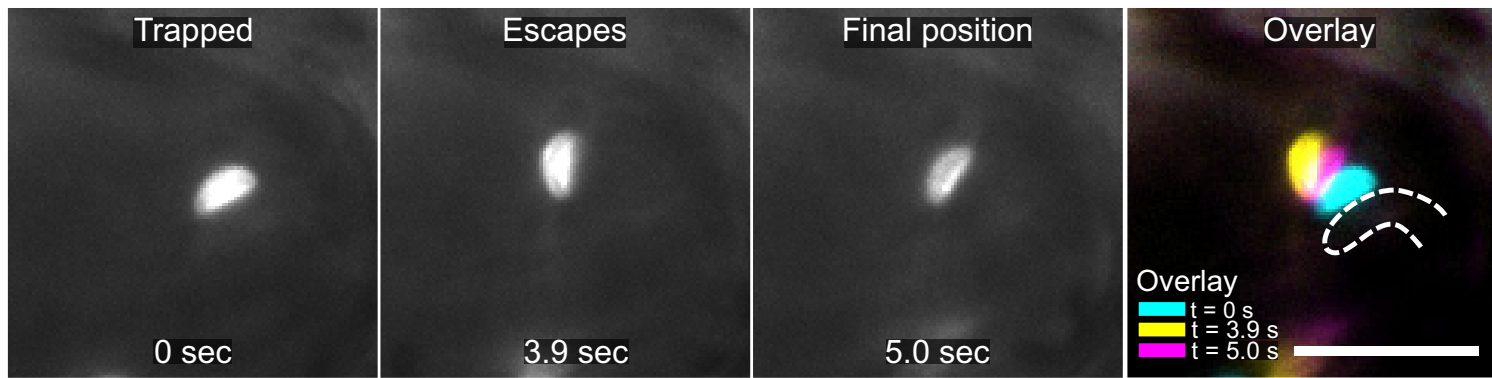

Figure 1. Chloroplasts accumulate at the host-pathogen interface during infection in an actin-dependent manner. (a) Maximum-projection confocal micrographs of Nicotiana benthamiana plants expressing GFP in chloroplast stroma (CpGFP), infected with Phytophthora infestans strain 88069td, showing chloroplast positioning around pathogen haustoria. Haustoria are marked by white arrowheads. (b) Maximum-projection confocal micrographs of wild-type N. benthamiana plants transiently expressing GFP actin chromobody, infected with $P$. infestans strain 88069td, following $24 \mathrm{~h}$ of treatment with water or $1 \mu \mathrm{M}$ of the chemical actin polymerization inhibitor latrunculin A (LatA). Haustoria are marked by white arrowheads. (c) Bar plots showing the percentage of chloroplast accumulation at haustoria following $24 \mathrm{~h}$ of treatment with water or $1 \mu \mathrm{M}$ LatA. Observations made in wild-type plants infected with $P$. infestans strain 88069td across four separate biological replicates, totaling 148 and 93 haustoria in the LatA and water conditions, respectively. Error bars show confidence intervals. ${ }^{*} P<0.05$, as determined by Fisher's exact test. (d) GFP channel in grayscale from a total internal fluorescence (TIRF) microscope. Time lapse showing the laser capture of a haustorium-associated chloroplast in the CpGFP plant where the automated trapping routine traps and attempts to move the chloroplast $10 \mu \mathrm{m}$. The chloroplast escapes the trap at $3.9 \mathrm{sec}$ before it springs back towards the original position at $5 \mathrm{sec}$. The dotted line shows the outline of the haustorium marked by RFP: REM1.3. Scale bars: $10 \mu \mathrm{m}$. 
group in regular day/night light-cycling conditions (lightdark). Live cell infection microscopy revealed no difference in chloroplast accumulation between the two lighting conditions (Figure S4). Collectively, these findings indicate that chloroplasts actively position themselves around haustoria during infection by $P$. infestans through a process that requires actin polymerization, but this process is not affected by changes in light conditions.

\section{Optical tweezers reveal the association between chloroplasts and haustoria}

Accumulating evidence points to the importance of organelle membrane contacts in response to various physiological or stress conditions (Helle et al., 2013; Liu and Li, 2019; Silva et al., 2020). To investigate the nature of the association of chloroplasts with haustoria/EHM, we used optical tweezers in combination with total internal fluorescence microscopy (TIRF) in CpGFP plants infected with the wildtype $P$. infestans 88069 and transiently expressing the EHM marker, RFP:REM1.3 (Bozkurt et al., 2014). The relocation of chloroplasts using optical tweezers by a distance of $>10 \mu \mathrm{m}$ was considered a successful movement. This threshold was set to ensure consistency between experiments; $10 \mu \mathrm{m}$ was chosen, as this distance was small enough to avoid side effects from moving the chloroplast towards the vacuole, such as pushing the chloroplast into the tonoplast.

Using optical tweezers, we successfully trapped and moved $17 \%$ of chloroplasts $(n=29)$ in non-haustoriated cells beyond the threshold using the automated trapping routine. In comparison, we were unable to trap and move any chloroplasts $(0 \%, n=18)$ neighboring haustoria past $10 \mu \mathrm{m}$, suggesting an association or connection between the chloroplasts and the EHM. Consistent with this finding, we also recorded instances where chloroplasts were initially pulled away from the EHM but before they passed the $10-\mu \mathrm{m}$ threshold they escaped the trap and sprang back towards their former position (22\%, $n=18$ ) (Figure 1d; Video Clip S8). These results suggest that chloroplasts may establish secure contacts with the EHM; however, further genetic and biochemical evidence supporting these findings are required to reach definitive conclusions.

\section{Chloroplasts can associate with haustoria independently of the host nucleus}

The relocation of the plant nucleus towards pathogen penetration sites was reported as a hallmark of plant focal immune responses (Griffis et al., 2014). Given recent reports showing the association of chloroplasts with the nucleus during plant stress responses (Caplan et al., 2015; Ding et al., 2019; Erickson et al., 2018), it is possible that chloroplasts are dragged to a haustorium along with the nucleus. However, this is unlikely because there is only a single nucleus per plant cell and $P$. infestans can form multiple haustoria that are in contact with chloroplasts within a single cell (Figure 1a).

Nevertheless, we quantified the numbers of haustoria associated with chloroplasts alone, compared with chloroplasts and nuclei together, to determine the extent to which chloroplast positioning around a haustorium correlates with the presence of the nucleus. To track the nucleus, we used leaf epidermal cells stably expressing GFP localized to the endoplasmic reticulum (ER-GFP herein) to visualize the silhouette of the nucleus (Figure 2). Confocal microscopy followed by the manual quantification of 463 haustoria revealed that $10 \%$ were associated with both a chloroplast and a plant nucleus (Figure 2a), whereas $26 \%$ were associated with just a chloroplast but not with a nucleus (Figure $2 \mathrm{~b}$ ). We observed that $62 \%$ of haustoria showed no association with a chloroplast or nucleus (Figure 2c). Very rarely (2\%), a haustorium was in contact with a nucleus without also being in contact with a chloroplast (Figure 2d). Nuclei in haustoriated cells that were not associated with a haustorium were still observed to be associated with chloroplasts (Figure S5), implying chloroplast-haustoria and chloroplast-nucleus associations are not mutually exclusive processes within a single, haustoriated cell.

Using the same methodology in Figure S1, we compared the frequency of actual chloroplast/nuclear accumulation at haustoria with hypothetical rates expected by chance encounter. Compared to mock haustoria, there were significantly higher cases of chloroplasts that associate with an actual haustorium (Figure S6). This observation was consistent when chloroplasts were associated with a nucleus or not (Figure S6a-b). However, we did not find any significant difference in association of nucleus alone to haustorium in mock versus real haustoria (Figure S6c). Taken together, these results suggest that chloroplast positioning towards haustoria can occur independently of nuclear migration, and that both chloroplast and nuclear accumulation at haustoria occurs at a greater frequency than is expected by chance.

\section{Chloroplasts alter their morphology and contact each other via the induction of stromules in response to infection}

During live cell imaging of CpGFP plants infected by $P$. infestans $88069 \mathrm{td}$, we also noted an increase in the frequency of stromules, from $4 \%$ in the mock-infected tissue to $19 \%$ in the infected tissue (Figure 3a,b). As previously reported, stromules varied in shape and size (Schattat et al., 2011), and some of these stromules extended towards and wrapped around haustoria (Figure 3c; Video Clip S9). Furthermore, stromules often extended between different chloroplasts, occasionally even bridging multiple haustoria (Figure S7; Video Clips S9 and S10). We also monitored stromulehaustorium associations by transiently expressing the EHM 
(a)

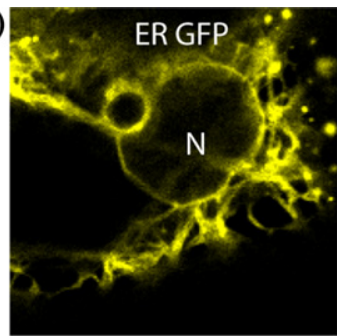

(b)

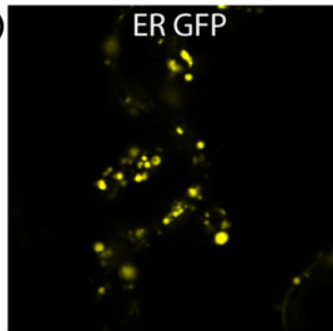

(c)

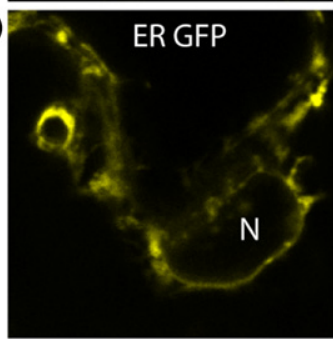

(d)

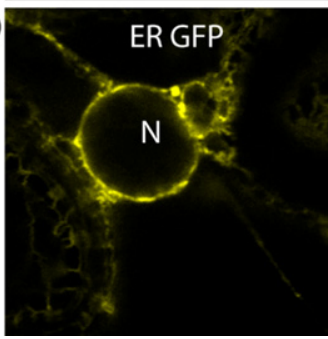

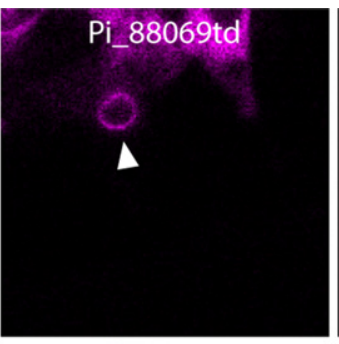
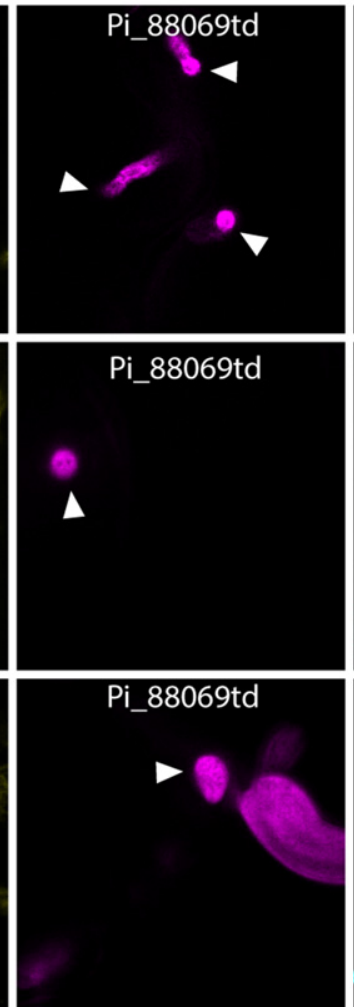
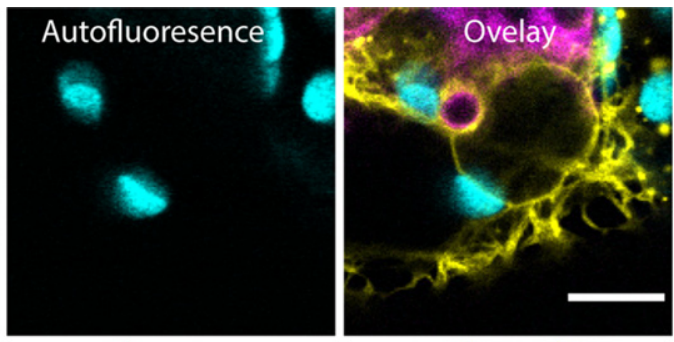

$10 \%$ of $\mathrm{N}=463$

haustoria

associated with

nucleus \&

chloroplast
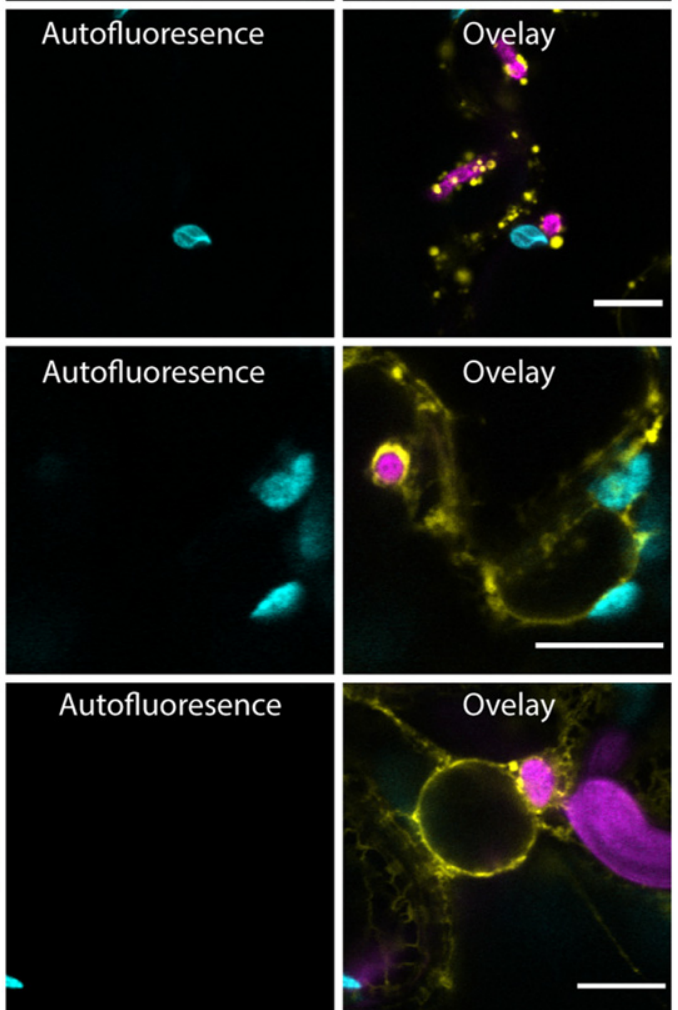

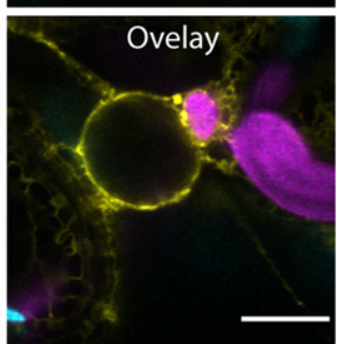

$62 \%$ of $\mathrm{N}=463$

haustoria not associated with chloroplasts or nucleus

$26 \%$ of $N=463$

haustoria

associated with

chloroplast only

\section{$2 \%$ of $\mathrm{N}=463$}

haustoria

associated with

nucleus only

Figure 2. Chloroplasts associate with haustoria, both with and without the host cell nucleus, during infection. Single-plane confocal micrographs of Nicotiana benthamiana plants expressing GFP in endoplasmic reticulum (ER-GFP), infected with Phytophthora infestans strain 88069td, showing chloroplast/nucleus positioning around pathogen haustoria. Haustoria are marked by white arrowheads; N, nucleus. Examples of the four combinations of chloroplast-nucleus association with haustoria and the percentage of each observation over 463 haustoria total. Scale bars: $10 \mu \mathrm{m}$. (a) Dual association of chloroplast and nucleus with haustoria. (b) Chloroplast alone at haustoria (no nucleus). (c) Haustoria unassociated with either chloroplast or nucleus. (d) Nucleus associated with haustoria alone.

marker protein RFP REM1.3 in leaves and infecting them with the wild-type $P$. infestans 88069 . We noted a close association of stromules with the EHM under these conditions (Figure 3c; Video Clip S11). These results are in agreement with the earlier reports that stromules can be induced by PAMPs (Caplan et al., 2015), and hint at defense-related roles of stromules, possibly through mediating chloroplast-chloroplast and chloroplast-EHM associations, as well as the previously reported chloroplast-nucleus associations (Caplan et al., 2015).

During our attempts to move chloroplasts in infected cells, we once attempted to move a chloroplast that was

Figure 3. Stromules are induced during infection with Phytophthora infestans. (a) Maximum-projection confocal micrographs of Nicotiana benthamiana plants expressing GFP in chloroplast stroma (CpGFP), showing the induction of stromules during infection with $P$. infestans 88069 td. (b) Scatter box plots of the percentage of chloroplasts with stromules for a given image in infected and uninfected samples (exemplified in panel a). Color of points show separate biological replicates. ${ }^{* * * * P} P 0.01$, as determined by Student's $t$-test. (c) Maximum-projection confocal micrographs of $N$. benthamiana plants expressing GFP in chloroplast stroma (CpGFP), infected with $P$. infestans, showing examples of stromules wrapping around haustoria. Haustoria are marked by white arrowheads. (i) Infection with wild-type $P$. infestans 88069 , plant cell transiently expressing RFP REM1.3 to mark the extrahaustorial membrane. (ii-iii) Infection with $P$. infestans 88069td. (d) GFP channel in grayscale from the TIRF microscope. Laser capture of chloroplast (*) linked by stromule to another chloroplast. When the trapped chloroplast moves, the linked chloroplast co-migrates. Scale bars: $10 \mu \mathrm{m}$. 
(a)

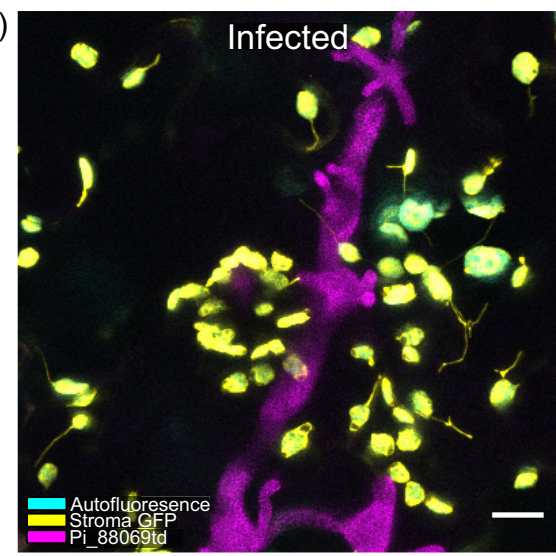

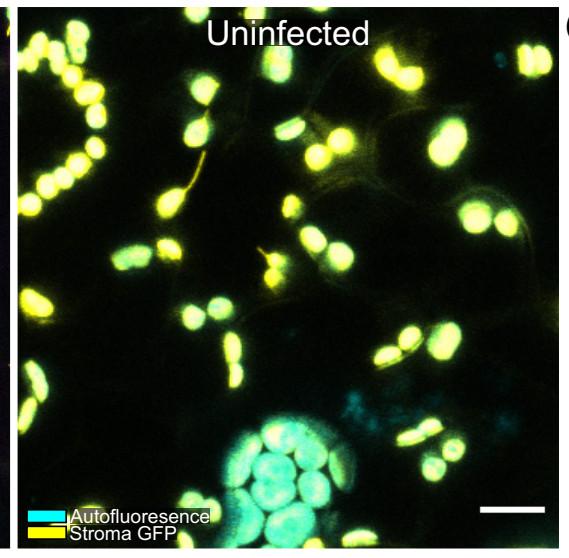

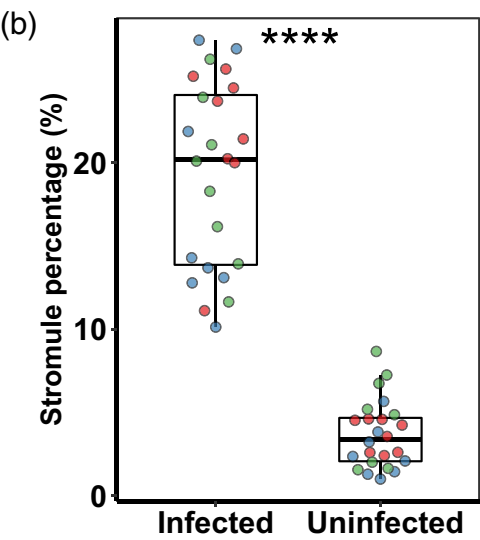

(c)

(i)

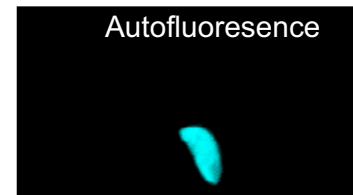

(ii)
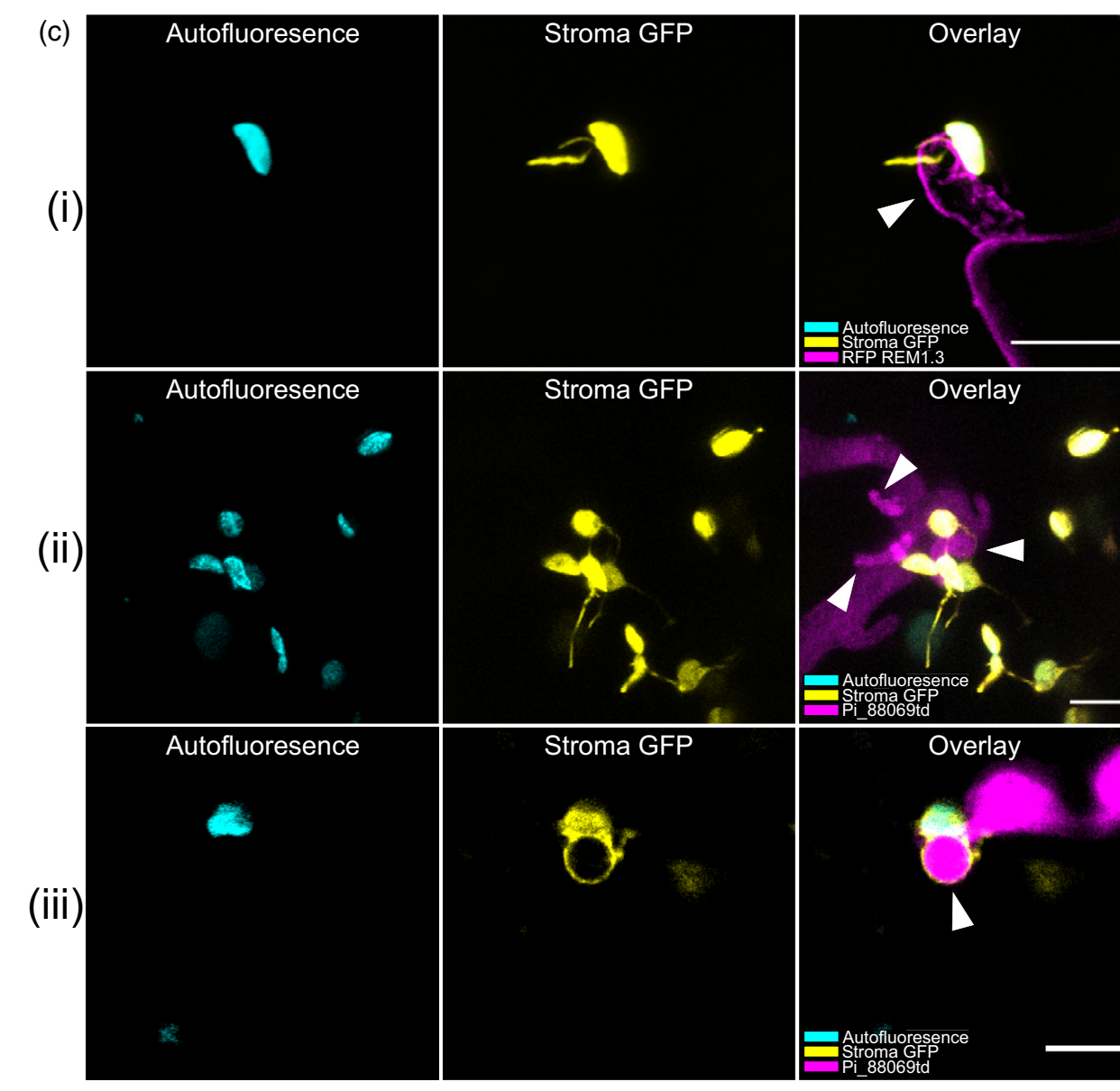

(d)
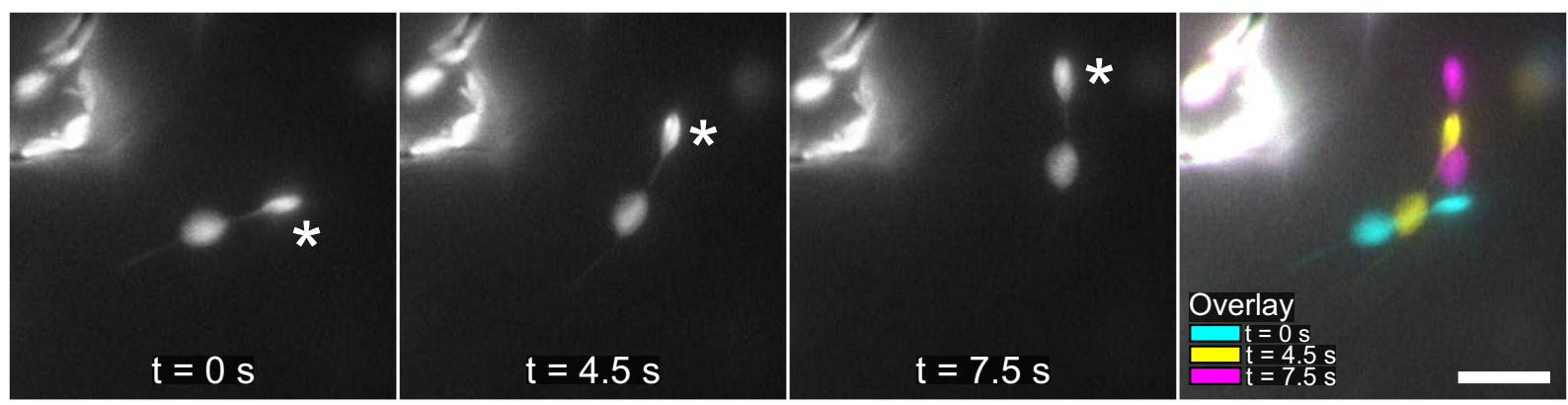
seemingly connected to another by a stromule like extension. Here, we observed the co-migration of the chloroplasts by moving only one of the pair (Figure $3 \mathrm{~d}$; Video Clip S12), indicating that chloroplasts may be linked by their stromules or that chloroplasts can follow one another when bridged by a stromule. Co-migration between nonstromule connected chloroplasts has also been observed (Caplan 2018). However, whether plastids can fuse to form (a)

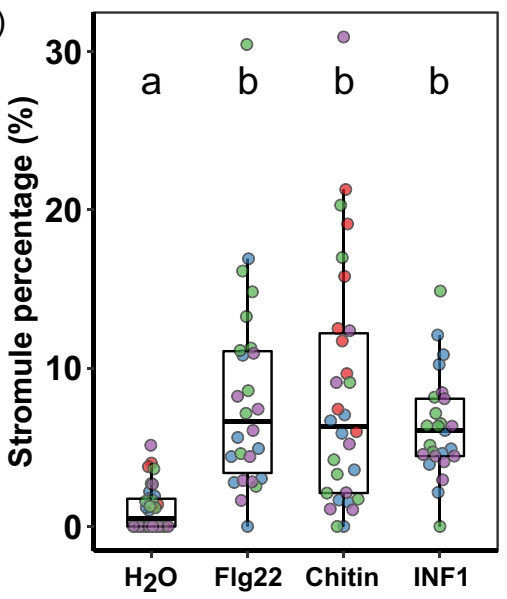

(d)

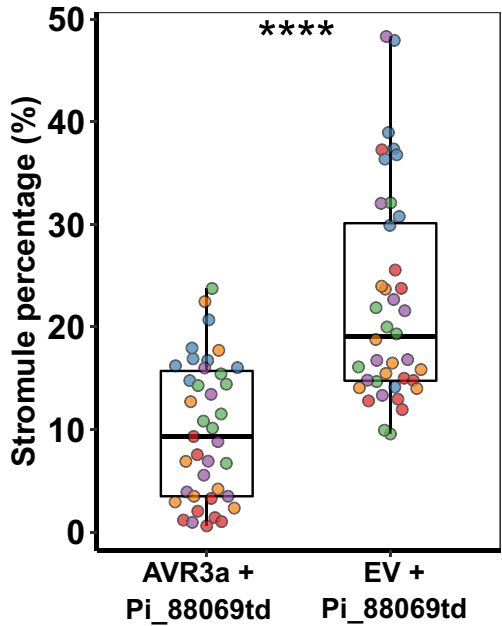

(g)

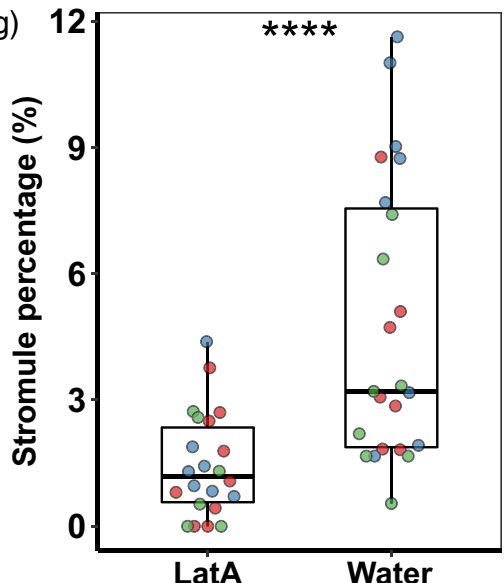

(b)

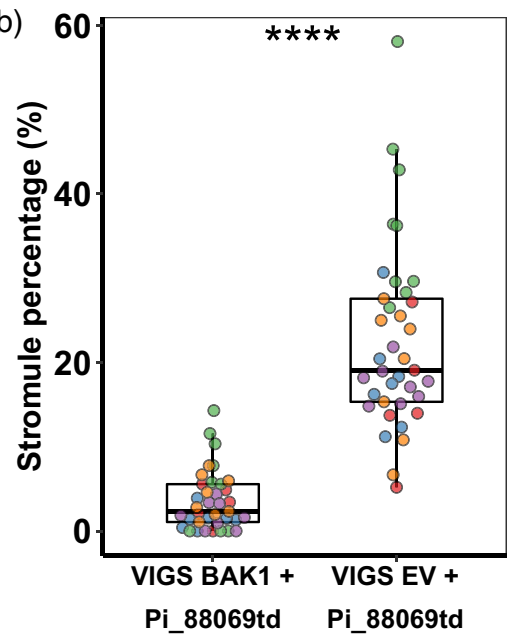

(e)

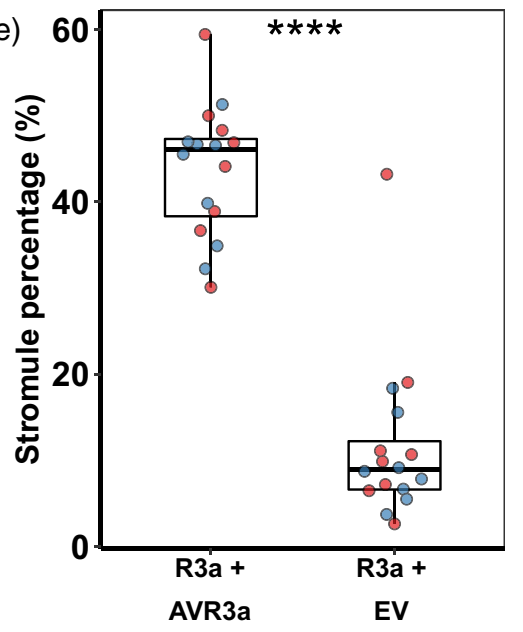

(h)

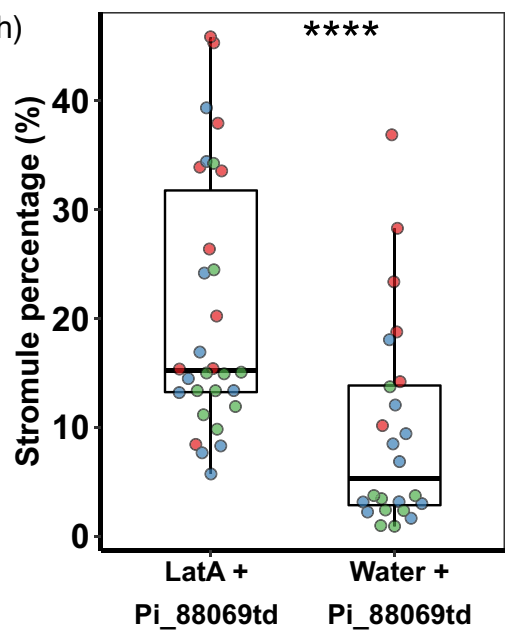

(c)

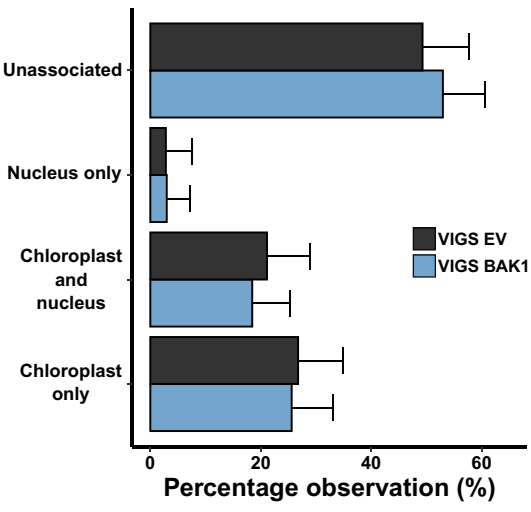

(f)

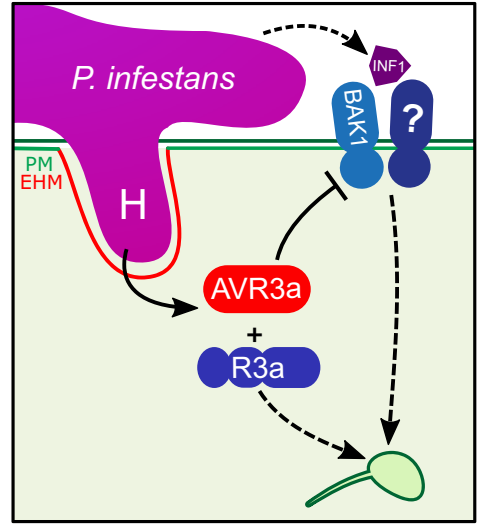


a continuous stromal compartment that enables macromolecule exchange thorough stromules remains under debate (Hanson and Hines, 2018; Schattat et al., 2015).

\section{Stromules are induced upon surface immune activation}

The PAMPs trigger a range of immune responses when recognized by surface-localized immune receptors (Jones and Dangl, 2006). Flg22, a peptide PAMP from bacterial flagellin, was previously shown to induce stromules (Caplan et al., 2015). We replicated this result with flg22 while also testing other elicitors: chitin, a polysaccharide PAMP of fungal microbes and arthropod pests; and INF1, an extracellular $P$. infestans protein that, unlike most PAMPs, elicits host cell death upon perception by the plant (Kamoun et al., 1998). Flg22, chitin and INF1 all induced stromules after $24 \mathrm{~h}$, compared with the water control (Figure 4a), suggesting that stromule production is a general response to a range of microbes. PAMP activity was confirmed by the detection of phosphorylated mitogenactivated kinases (MAPKs) by Western blot (Figure S8).

BAK1 is a surface-localized co-receptor that mediates immune signaling in co-operation with various patternrecognition receptors to perceive PAMPs (Chaparro-Garcia et al., 2011; Heese et al., 2007). Therefore, we monitored stromule formation upon the systemic silencing of $B A K 1$ by virus-induced gene silencing (VIGS) in CpGFP plants (Figure S9) during infection (Figure 4b). We measured a substantial decrease in infection-triggered stromule induction following $B A K 1$ silencing $(4 \%, n=37$ images quantified) compared with control silencing $(23 \%, n=37$ images quantified), corroborating the induction of stromules by PAMPs and suggesting that PAMPs from $P$. infestans induce stromules during infection.

Extending this, we tested whether the silencing of $B A K 1$ through VIGS would alter chloroplast and nucleus accumulation at the haustorium. To do this, we used live cell infection microscopy of $B A K 1$-silenced ER-GFP plants. We found no significant difference in chloroplast and/or nuclear accumulation at haustoria during infection, compared with silencing controls ( $n=168$ and $n=142$ haustoria, respectively) (Figure 4c). This indicates that BAK1-dependent signaling for stromule induction is independent from that of chloroplast accumulation at the host-pathogen interface.

To complement the $B A K 1$-silencing phenotypes on stromule formation, we then tested whether AVR3a, a host-translocated effector of $P$. infestans that suppresses BAK1-mediated immune signaling (Chaparro-Garcia et al., 2011, 2015), can perturb pathogen-induced stromule development. Following transient expression of AVR3a, stromule formation during infection decreased significantly, from 23 to $10 \%$ (Figure $4 \mathrm{~d}$ ). This indicates that pathogen effectors can perturb stromule induction, further supporting a defense-related role of stromules.

The functionality of AVR3a was confirmed by observing cell death when transiently co-expressed with the cognate R3a NLR immune receptor (Figure S10). We also observed that in the absence of the pathogen, transient coexpression of AVR3a with R3a led to an induction of stromules compared with the control, where R3a was transiently co-expressed with EV instead of AVR3a (Figure 4e). This points towards the induction of stromules by HR cell death triggered by effector recognition, despite the functionality of the effector in suppressing stromules when it goes undetected by the plant. Taken together, these results show the induction of stromules by both PAMP triggered immunity or effector triggered immunity, and the suppression of stromules by pathogen effectors that target PTI sig naling (Figure 4f).

Following this, we investigated the effect of actin disruption on stromule frequency. Various chemical actin inhibitors have been shown to reduce stromule frequency in epidermal plastids (Kwok and Hanson, 2003; Natesan et al., 2009). Supporting this, we found that after $24 \mathrm{~h}$ of treatment with $1 \mu \mathrm{M}$ LatA, the epidermal chloroplasts of CpGFP plants had significantly fewer stromules than after control

\footnotetext{
Figure 4. Stromule induction through immune recognition. (a) Scatter box plots of the percentage of chloroplasts with stromules for a given image following treatment of CpGFP plants for $24 \mathrm{~h}$ with water, flg22, chitin or INF1. Colors of the points show separate biological replicates. Letters mark significance groups (where $\alpha=0.01$ ), as determined by Dunn's test with $P$ values adjusted by Bonferroni correction. (b) Scatter box plots of the percentage of chloroplasts with stromules for a given image during infection of CpGFP plants by Phytophthora infestans strain 88069td in VIGS BAK1 and VIGS EV (control) plants. Colors of the points show separate biological replicates. $* * * * P<0.01$, as determined by Wilcoxon test. (c) Bar plots showing percentage of chloroplast/nuclear accumulation at haustoria in VIGS BAK1 plants, compared with VIGS EV plants. Observations made in ER-GFP plants infected with $P$. infestans strain 88069td, across three separate biological replicates, totaling 168 and 142 haustoria in the VIGS BAK1 and VIGS EV conditions respectively. Error bars show confidence intervals. No statistically significant difference detected $(\alpha=0.05)$, as determined by Fisher's exact test. (d) Scatter box plots of the percentage of chloroplasts with stromules for a given image during infection of CpGFP plants by $P$. infestans strain 88069td during transient plant cell expression of either AVR3a or the EV control. The colors of the points show separate biological replicates. $* * * *<0.01$, as determined by Wilcoxon test. (e) Scatter box plots of the percentage of chloroplasts with stromules for a given image following transient co-expression of either R3a with AVR3a or R3a with an EV control. The colors of the points show separate biological replicates. ${ }^{* * * *} P<0.01$, as determined by Wilcoxon test. (f) Model of possible immune-related signaling pathways that can lead to stromule induction, evidenced by the data shown in (a), (b), (d) and (e). (g) Scatter box plots of the percentage of chloroplasts with stromules for a given image following $24 \mathrm{~h}$ of treatment with $1 \mu \mathrm{M}$ LatA or water control. Color of point shows separate biological replicate. Asterisks denote $P<0.01$ as determined by Wilcoxon test. (h). Scatter box-plots of the percentage of chloroplasts with stromules for a given image in CpGFP plants infected with $P$. infestans strain 88069 td following $24 \mathrm{~h}$ treatment with $1 \mu \mathrm{M}$ latrunculin $\mathrm{A}$ (LatA) or water control (image 4 days post-infection). The colors of the points show separate biological replicates. $* * * * P<0.01$, as determined by Wilcoxon test.
} 
treatments (Figure $4 \mathrm{~g}$ ), confirming the role of actin stromule maintenance. We next tested the effect of this treatment in tissue infected with $P$. infestans strain 88069td. To our surprise, we found that the LatA treatment caused the opposite effect in infected tissue compared with uninfected tissue, consistently boosting stromule levels compared with the control treatment (Figure $4 \mathrm{~h}$ ), suggesting a more complex role of actin in infected related stromules.

To complement this, we investigated the role of microtubules in stromule frequency by treating CpGFP plants with $100 \mu \mathrm{M}$ of the chemical microtubule disruptor oryzalin. Following 24 or $3 \mathrm{~h}$ of treatment, we found no significant difference in stromule frequency in uninfected or infected tissues (Figure S3b,c,f,g). The role of microtubules in guiding stromule extension has been shown concurrently and independently through the use of rigorous observational microscopy (Erickson et al., 2018; Kumar et al., 2018); however, the effect of chemical microtubule disruption on stromule frequency has shown differing results (Kumar et al., 2018; Natesan et al., 2009), probably linked to differences in treatment concentrations and timing, as well as the cell types observed.

\section{HyPer ROS reporter displays increased reactivity in chloroplasts upon infection}

Previously, Caplan et al. (2015) proposed that the close proximity of chloroplasts to the plant nucleus could allow for ROS transfer to the nucleus via stromules. We reasoned that chloroplast positioning around the haustorium may serve to increase localized ROS production by chloroplasts at the site of infection. To test this, we visualized ROS in live cell infection using the HyPer ROS sensor fused to the chloroplast transit peptide of Arabidopsis thaliana RecA, cTP-HyPer herein (Caplan et al., 2015). In the presence of ROS, the ratio between emission intensity from 405- and 488-nm excitation changes, gives a detectable read out of ROS from confocal microscopy (Belousov et al., 2006).

The epidermal chloroplasts of infected cells showed noticeably more signal than those of uninfected cells (Figure 5a). However, within a single haustoriated cell, we (a)
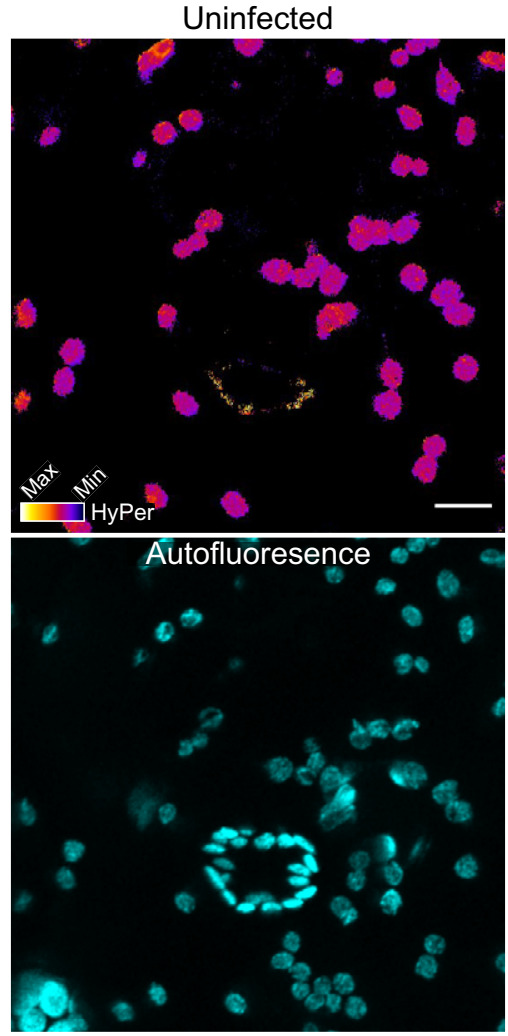
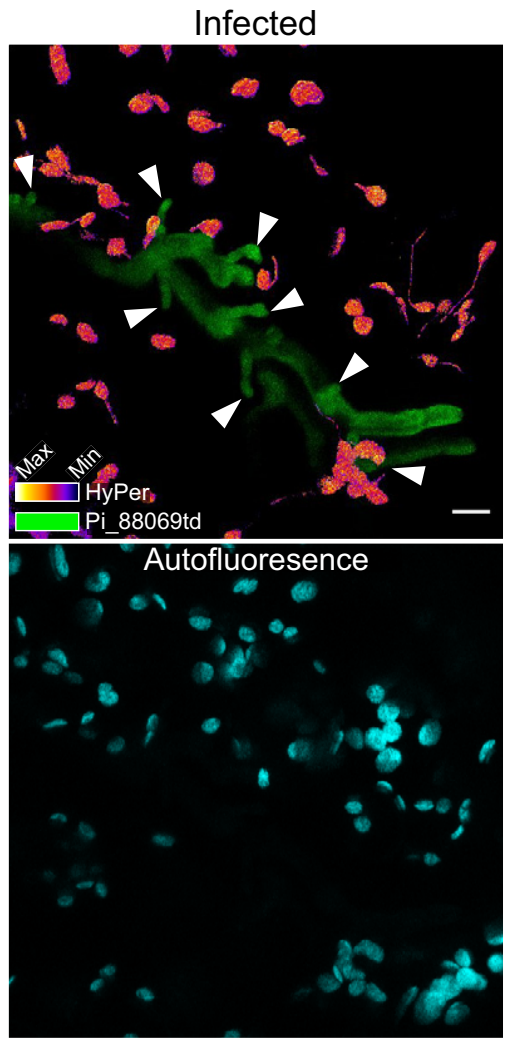

(b)

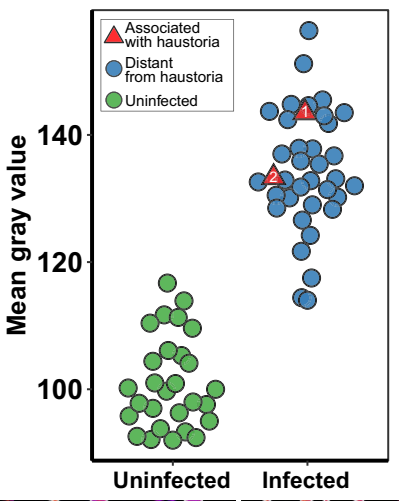

(c)

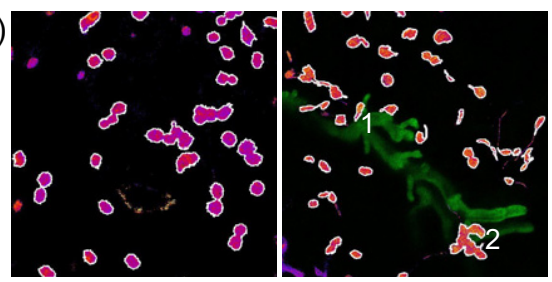

ROI overlay

Figure 5. Chloroplasts increase reactive oxygen species (ROS) production during infection. (a) Maximum-projection confocal micrographs of wild-type Nicotiana benthamiana plants transiently expressing the plastid-localized cTP-HyPer ROS sensor, with and without live infection by Phytophthora infestans strain 88069td. Visual representation of the cTP-HyPer signal intensity ratio between excitation by 405- and 488-nm lasers, shown with the IMAGEJ 'Fire' Look Up Table (LUT). Haustoria are marked by white arrowheads. Scale bars: $10 \mu \mathrm{m}$. All confocal settings were kept identical between infected and uninfected plants during acquisition. (b) Quantification of the cTP-HyPer signal in the chloroplasts shown in (a), where the mean gray value (i.e. signal intensity) is displayed. Regions of interest (ROls) measured and plotted are shown in (c). ROls capturing chloroplasts associated with haustoria have been plotted as red triangles. (c) ROIs quantified for mean gray value in (b), shown as a solid white line. Chloroplasts associated with a haustorium have been numbered (1 and 2 ), and correspond to the numbered points shown in (b). 
could not see a discernable difference in ROS signal between the different plastids, including between those associated and those unassociated with haustoria (Figure 5a). The mean signal intensity was measured to show the ROS signal levels quantitatively as well as qualitatively (Figure 5b), targeting regions of interest containing chloroplasts (Figure $5 \mathrm{c}$ ). We speculate that chloroplast ROS production and visualization may be highly variable as a result of differences in the stages of infection and the changes in the microenvironment, as well as potential manipulation by effector secreted by the pathogen.

\section{DISCUSSION}

Here, we demonstrate that during host colonization by $P$. infestans, chloroplasts accumulate at the pathogen interface (Figures 1,2) and alter their morphology through the induction of stromules (Figures 3,4). Additionally, we show that nuclei are almost exclusively localized to haustoria in the company of a chloroplast, but that chloroplasts can accumulate independently (Figure 2). Stromules occasionally embrace the EHM and facilitate chloroplastchloroplast contact, forming dynamic organelle clusters around the pathogen interface (Figures $3 \mathrm{c}$ and S7) that are reminiscent of mitochondrial networks, with poorly understood functions (Hoitzing et al., 2015). Notably, infectiontriggered stromule development relies on surface immune signaling, whereas the pathogen can subvert this process, interfering remotely with these pathways (Figure 4f). Our results implicate chloroplasts in cell polarization upon pathogen attack and point to more complex functions of these organelles in plant-pathogen interactions.

\section{How do chloroplasts position themselves at the pathogen interface?}

It is well established that chloroplasts alter their subcellular localization in response to light in an actin-dependent manner (Kadota et al., 2009; Wada and Kong, 2018); additionally, actin plays a role as an anchor point for chloroplasts to other cellular compartments, such as the nucleus (Kumar et al., 2018). We found that infection-induced chloroplast positioning around the haustorium relies, to an extent, on actin polymerization (Figure $1 b, c)$, whereas the process is not affected by light conditions (Figure S4). Previous studies have shown that modulation of the actin cytoskeleton can affect cell penetration by filamentous plant pathogens (Kobayashi and Hakuno, 2003; Tang et al., 2016), and others have noted the reorganization of actin around the site of pathogen penetration (Opalski et al., 2005). Our finding, that actin polymerization inhibitor LatA reduces chloroplast positioning at haustoria (Figure 1c), is in agreement with previous studies that implicate actin in the focal immune response, as well as the known importance of actin in chloroplast movement (Higa et al., 2014; Suetsugu et al., 2016; Suetsugu and Wada, 2016).
However, the use of LatA to disrupt actin polymerization is not refined enough to distinguish whether impaired chloroplast-haustoria localization results from a disruption of actin-mediated chloroplast movement and/or disruption to actin-mediated chloroplast anchoring. Although this is a promising first step in altering chloroplast positioning during infection, we also heed caution regarding the use of pharmacological treatment for live infection systems, with potential off-target effects in both host and pathogen. Ideally, work following on from this would move towards using targeted genetic approaches or identifying effectors that can target this process more specifically.

Additionally, we monitored chloroplast association to haustoria in the context of nuclear association (Figure 2). Although the movement of nuclei towards plant-pathogen interfaces appears to be under varying spatiotemporal dynamics in different pathosystems (Griffis et al., 2014; Scheler et al., 2016), evidence suggest that this process may contribute to plant immunity (Daniel and Guest, 2006). However, nuclear movement during cell penetration by filamentous microbes is not exclusively unidirectional, with the nucleus moving first towards and then away from penetration sites in many interactions (Genre et al., 2005; Schmelzer, 2002). Our results regarding the accumulation of nuclei provide a snapshot at a single time point of infection, and therefore our conclusions are derived from the observed state of the nucleus at that time point, not considering whether the nucleus is in the process of moving towards or away from the haustorium. Further dissection of specific nuclear movement components over extended time courses is required to address the intricacies and impact of nuclear movement in plant focal immunity.

\section{Why do chloroplasts alter their morphology during infection?}

Our findings on PAMP induction of stromules (Figure 4a) support and expand upon those reported by Caplan et al. (2015), who previously showed that flg22 can induce stromules. The reduction of stromules during infection by the systemic silencing of $B A K 1$ (Figure 4b) suggest that a major subpopulation of stromules (if not all) induced during infection rely on BAK1-mediated immune signaling initiated at the cell surface. Here, we used AVR3a, an effector protein, as a tool to cross-examine the role of BAK1 (Figure $4 d$ ) because it is known to suppress BAK1-mediated defense signaling (Chaparro-Garcia et al., 2011, 2015). Furthermore, we hypothesize that other effector proteins are probably able to inhibit stromules indirectly by targeting similar cell surface signaling pathways.

Interestingly, BAK1 silencing did not affect the positioning of chloroplasts and/or the nucleus around haustoria (Figure 4c), hinting at differential signaling pathways between stromules and organelle positioning. As of yet, we do not know what signaling takes place to re-route 
plant defenses towards the host-pathogen interface. The decoupling of stromule induction and chloroplast accumulation at haustoria is further supported by our time-lapse imaging, which shows that the accumulation of chloroplasts at haustoria can occur independently of stromules, although we observe both stromule-led and stromuleindependent movement towards haustoria (Video Clips S3 and S4). This is in keeping with observations by Kumar et al. (2018), who found that chloroplast movement is mostly, but not exclusively, stromule directed.

The induction of stromules by immune signaling during pathogen attack strongly points to defense-related functions of these tubular organelle extensions. But how could stromules contribute to immunity? Although our understanding of stromules is still limited by a lack of stromulespecific inhibitors/inducers, a model of stromules as signaling conduits is emerging. Caplan et al. (2015) suggest that the increased surface area provided by a stromule aids in the transfer of chloroplast synthesized pro-defense molecules to the cytosol and nucleus where they function. The increase in chloroplast-nucleus contact, facilitated by stromules, and triggered during an HR response, is thought to amplify the progression of HR in a positive feedback loop. Similarly, a more recent study tracked the spatiotemporal redox state of chloroplasts, and stromule induction, in Solanum tuberosum (potato) during challenge with potato virus $Y$ (Lukan et al., 2021). Lukan et al. (2021) conclude that, in this pathosystem, stromules are involved in signaling on the virus multiplication front.

We observed that chloroplasts establish network-like interactions via stromules (Figure S7), and that some chloroplasts intimately associate with the haustorium interface through stromules (Figure 3c). These chloroplast clusters and stromule extensions around the haustorium could plausibly aid the coordination of defense-related functions of chloroplasts by, for instance, mediating the deployment of pro-defense molecules at the pathogen interface. Our findings further support the notion that stromules are induced to contribute to pathogen defense (Caplan et al., 2015; Erickson et al., 2018), but the function of stromules still remains to be determined (Hanson and Hines, 2018).

\section{Stromules and the cytoskeleton}

Disruption of cytoskeletal elements has been shown to affect stromule frequency, velocity and length. Treatment of Nicotiana tabacum (tobacco) with the chemical actin inhibitor cytochalasin $D$ reduces stromule frequency in epidermal plastids (Kwok and Hanson, 2003); furthermore, $10 \mu \mathrm{M}$ latrunculin $\mathrm{B}$ significantly reduces stromule frequency after $60 \mathrm{~min}$ in tobacco epidermal cells (Natesan et al., 2009). Here, we show that treatment with $1 \mu \mathrm{M}$ LatA also significantly reduces stromule induction in otherwise untreated epidermal cells (Figure 4g). However, we unexpectedly found that during infection, LatA treatment had the opposite effect, causing an increase in stromule induction (Figure 4h). One hypothesis we have regarding this inverse phenotype relates to the role of actin as an anchoring point for the chloroplast to the plasma membrane and nucleus (Kumar et al., 2018; Oikawa et al., 2008). Pathogen challenge could cause an increase in chloroplast activity and movement, as suggested by the data presented in Figure 1. Actin-mediated chloroplast anchoring might reduce chloroplast movement and stromules in normal infected conditions; when this is disrupted by actin-inhibiting treatments, actin-independent movement and stromule production increases through the lack of anchoring. In this scenario, our previous results showing that LatA reduces chloroplast-haustoria association would be best explained by a loss of actin-mediated chloroplast anchoring to the sites of intracellular pathogen penetration. Further experimentation is essential to test these hypotheses, such as the concurrent treatment of infected and uninfected tissues with both an actin and a microtubule inhibitor, ideally with concurrent imaging of both actin microfilaments and microtubules.

Although our experiments showed that treatment with $100 \mu \mathrm{M}$ oryzalin did not affect stromule induction in infected and uninfected tissues (Figure S3b,c,f,g), we cannot conclusively dispel the role of microtubules in stromule induction and maintenance, especially considering that the efficacy of the chemical had waned by $24 \mathrm{~h}$ (Figure S3d,e). Kumar et al. (2018) found that treatment with $300 \mu \mathrm{M}$ oryzalin reduced stromule frequency after $15 \mathrm{~min}$ in $N$. benthamiana epidermal cells. In tobacco epidermal cells, treatment with $36 \mu \mathrm{M}$ oryzalin had no effect on stromule frequency over a period of $75 \mathrm{~min}$ (Natesan et al., 2009). As noted by Kumar et al. (2018), differences in tissue types and inhibitor concentrations are likely to be behind the inconsistencies in observed effects; we would add that the timing of treatments used before assessing stromule frequency is also likely to contribute to variation in the perceived effect.

\section{Right time, right place: Chloroplast position at pathogen interface is a host defense or pathogen strategy?}

It is unclear whether the association of chloroplasts with the haustoria of $P$. infestans is a plant defense or a pathogen virulence strategy. Given the arsenal of immune chemicals produced by chloroplasts, it is plausible that their presence at haustoria may enhance the effectiveness of their deployment; furthermore, chloroplast association with haustoria could induce further immune signaling, possibly through retrograde (chloroplast-to-nucleus) signaling. However, we cannot discount the possibility that chloroplasts accumulate at haustoria to the benefit of the pathogen, perhaps serving to nourish the parasite. Specific, genetic strategies that impair chloroplast positioning around the haustorium are necessary to reach definitive conclusions on this. Expanding this work to other pathosystems, particularly those distantly related or those with a different lifestyle (i.e. completely 
biotrophic), as well as using alternative strains of $P$. infestans with different effector repertoires would also help resolve the physiological and evolutionary relevance of this observed response. We believe this work will lay the foundation for future studies regarding chloroplast movement towards, and association with, intracellular pathogen structures. By further defining the role of surface immune signaling in stromule induction and showing how effectors can be used to manipulate this process, we believe these tools will help to accelerate research into stromule function and signaling pathways.

\section{EXPERIMENTAL PROCEDURES}

\section{Biological material}

Nicotiana benthamiana plants were grown in a growth chamber at $25^{\circ} \mathrm{C}$ under high light intensity (16-h day/8-h dark photoperiod). Transplastomic GFP-expressing $N$. benthamiana plants, accumulating GFP in the chloroplast stroma (Stegemann et al., 2012), and transgenic GFP-expressing $N$. benthamiana plants, accumulating GFP in the endoplasmic reticulum, were maintained in the same conditions as wild-type $N$. benthamiana. Phytophthora infestans isolate 88069 (Van West et al., 1998) and 88069td (Whisson et al., 2007), a transgenic strain expressing the red fluorescent marker tandem dimer RFP (tdTomato), were used. Both isolates were cultured on plates with rye sucrose agar (RSA) for $12-16$ days at $18^{\circ} \mathrm{C}$ in the dark, as described elsewhere (Song et al., 2009), prior to use for infection in $N$. benthamiana.

\section{Plasmid constructs}

The following constructs used in this study have been described previously: RFP:REM1.3 (Bozkurt et al., 2014); R3a (ChaparroGarcia et al., 2015); AVR3a cloned in pICSL86977 was provided by TSLSynBio; GFP Actin chromobody (Rocchetti et al., 2014). Silencing construct TRV2-BAK1 was kindly provided by The Sainsbury Laboratory (Chaparro-Garcia et al., 2011). The RecA-cTP HyPer construct was kindly provided by Prof. Savithramma DineshKumar (Caplan et al., 2015).

\section{Transient gene-expression assays in $\mathbf{N}$. benthamiana}

Agrobacterium tumefaciens strain GV3101 (Hellens et al., 2000) carrying T-DNA constructs was used to mediate transient gene expression (referred to in the text as transient expression) into 34-week-old $N$. benthamiana leaves, as previously described (Bozkurt et al., 2011, 2014). Briefly, overnight cultures of transformed A. tumefaciens were washed and harvested with $1500 \mu \mathrm{L}$ autoclaved $\mathrm{dH}_{2} \mathrm{O}$ by centrifugation at $1500 \mathrm{~g}$ twice and resuspended in agroinfiltration buffer (10 mM 2- $\mathrm{N}$-morpholino-ethanesulfonic acid hydrate (MES hydrate), $10 \mathrm{mM} \mathrm{MgCl}_{2}, \mathrm{pH}$ 5.7). For the transient expression assays, each $A$. tumefaciens construct was mixed in agroinfiltration buffer to achieve the desired final $\mathrm{OD}_{600}$ : for GFP actin, $\mathrm{OD}_{600}=0.05$; for AVR3a, $\mathrm{R3}$ a and $E V, \mathrm{OD}_{600}=0.3$; for RFP REM1.2, $\mathrm{OD}_{600}=0.3$; for cTP-HyPer, $\mathrm{OD}_{600}=0.2$. The $P$. infestans inoculations were performed $4-24 \mathrm{~h}$ after infiltrations, if at all.

\section{Virus-induced gene silencing (VIGS)}

Agrobacterium was prepared as described above carrying TRV1 and the appropriate TRV2 construct and mixed to a final $\mathrm{OD}_{600}$ of 0.4 or 0.2 , respectively, in agroinfiltration buffer supplemented with $100 \mu \mathrm{M}$ acetosyringone (Sigma-Aldrich, https://www.sigmaa
Idrich.com) and left in the dark for $2 \mathrm{~h}$ prior to infiltration to stimulate virulence. Then, 14-day-old $N$. benthamiana seedlings were infiltrated in cotyledons and any true leaves that had emerged. Nicotiana benthamiana plants were infiltrated with TRV1 and TRV2-BAK1 for BAK1 silencing, and with TRV1 and TRV2-EV for the empty vector control. TRV2 containing the $N$. benthamiana sulfur (Su) gene fragment (TRV2-NbSU) was used as a positive control to indicate viral spread. Plants were left to grow under standard conditions until experiments could be carried out 4 weeks later.

\section{Phytophthora infestans infection}

Zoospores were harvested from sporangia by the addition of cold distilled water and collected after $2 \mathrm{~h}$ of incubation at $4^{\circ} \mathrm{C}$, adjusting the dilution to 50000 spores $\mathrm{ml}^{-1}$. Infections were performed by the addition of $10 \mu \mathrm{l}$ of zoospore droplets to the abaxial side of the leaf. The infected leaves were maintained in plastic boxes on damp paper towels at $18^{\circ} \mathrm{C}$ under $16-\mathrm{h}$ day/8-h night conditions (except in the dark/dark experimental conditions, where there was no day period; Figure S4).

\section{PAMP treatments}

Flg22 and INF1 purified peptides were provided by The Sainsbury Laboratory (http://www.tsl.ac.uk). Chitin was prepared from powdered shrimp shell (Sigma-Aldrich) following the manufacturer's protocol. Working concentrations of $1 \mu \mathrm{M}$ (flg22 and INF1) and $100 \mu \mathrm{g} \mathrm{ml}^{-1}$ (chitin) were used, unless otherwise stated. Dilutions were made in water and infiltrated into the underside of leaves using a needle-free syringe.

\section{LatA and oryzalin treatment}

Latrunculin A (abcam, https://www.abcam.com) was diluted to a stock concentration of $100 \mu \mathrm{M}$ in $100 \%$ DMSO. The water control was prepared with a matching final concentration of DMSO $(\mathrm{v} / \mathrm{v})$. Oryzalin was solubilized in methanol and used at a working concentration of $100 \mu \mathrm{M}$, with water controls being prepared with matching final concentrations of methanol $(1.66 \% \mathrm{v} / \mathrm{v})$. LatA, oryzalin and controls were infiltrated into leaf tissue by needlefree syringe, $24 \mathrm{~h}$ before microscopy.

\section{Visualization of chloroplast ROS}

Live cell imaging of chloroplast ROS was imaged using the HyPer ROS sensor (Belousov et al., 2006) fused to the chloroplast transit peptide of $A$. thaliana RecA. This ROS-sensitive fluorescencebased marker is imaged by fast line switching between $405-\mathrm{nm}$ (channel 1) and 488-nm excitation (channel 2), detecting emission in the range of $491-543 \mathrm{~nm}$. The ratio between emission from channel 1 and channel 2 gives the final signal by dividing the signal from channel 2 by the signal from channel 1 in IMAGEJ using the inbuilt 'Math' functions (Schneider et al., 2012). The look-up table was set to 'Fire' in IMAGEJ for the improved visualization of intensity and the HyPer signal channel for all images was adjusted in brightness by setting the maximum displayed value to 300 (for 32-bit images). The resulting HyPer signal was also quantified in chloroplast regions of interest (ROIs) by simply measuring the mean gray value in each $\mathrm{ROI}$.

\section{RT-PCR assay}

A 60-mg sample of leaf tissue was excised from 5-week-old leaves (VIGS experiments) and frozen in liquid $\mathrm{N}_{2}$. RNA was extracted from the leaf tissue using the Plant RNA Isolation Mini Kit Protocol (Agilent Technologies, https://www.agilent.com). RNA quality and 
concentration was measured using a NanoDrop ${ }^{\mathrm{TM}}$ Lite Spectrophotometer (ThermoFisher Scientific, https://www.thermofisher.com). cDNA was synthesized using as a $2 \mu \mathrm{g}$ template of RNA following the SuperScript II RT protocol (Invitrogen, now ThermoFisher Scientific). To amplify the cDNA, a standard PCR (RT-PCR) was then performed using DreamTaq DNA polymerase $\left(5 \mathrm{U} \mathrm{\mu l}^{-1}\right.$ ) (ThermoFisher Scientific). VIGS BAK1 silencing was confirmed as previously described (Chaparro-Garcia et al., 2011).

\section{Confocal microscopy}

All microscopy analyses were performed on live $N$. benthamiana epidermal cells 2-6 days post-agroinfiltration and infection. Leaf discs were excised and imaged on either an SP5 or SP8 resonant inverted confocal microscope (Leica Microsystems, https://www.le ica-microsystems.com) using a $63 \times$ or $40 \times$, respectively, $1.2 \mathrm{NA}$ Plan-Apochromat water immersion objective. Specific excitation wavelengths and filters for emission spectra were set as described previously (Koh et al., 2005). The Argon laser excitation was set to $488 \mathrm{~nm}$ and the Helium-Neon laser was set to $543 \mathrm{~nm}$ and their fluorescent emissions were detected at $495-550$ and $570-620 \mathrm{~nm}$ to visualize GFP and RFP fluorescence, respectively. To avoid bleed-through from different fluorophores, images were acquired using sequential scanning and maximum-intensity projections were created from the $Z$-stacks. 3D images and videos were generated with confocal files in 12-bit TIFF format imported into NISELEMENTS 4.50 (Nikon Instruments Inc., https://www.microscope.hea Ithcare.nikon.com) and processed with ADVANCED DENOISING. Videos were made using the 'Volume View' and 'Video Maker' modules.

\section{Optical trapping set-up}

The optical trap for chloroplast/stromule capture was set up as described by Sparkes et al. (2018: Chapter 13). An optical trap with a two-channel TIRF microscope (TIRF-M) was combined with a Nikon Ti-U inverted microscope. Optical trapping was performed using a near-infrared trapping laser at $1070 \mathrm{~nm}$ using a Nikon $100 \times$, oil immersion, NA 1.49 TIRF objective lens. For GFP and RFP, chromophores fused to the proteins of interest were excited using 488- and 561-nm laser diodes, respectively. Their fluorescent emissions were detected using two electron multiplying charge-coupled device (EMCCD) cameras (iXon; Andor Technology, https://andor.oxinst.com). The sample (approx. $5 \mathrm{~mm}^{2}$ of leaf tissue) was mounted on a computer-controlled variable speed stepper motor stage (Märzhäuser Wetzlar $\mathrm{GmbH}$, https://www.ma rzhauser.com). The associated computer-controlled hardware was interfaced using LABVIEW (National Instruments https://www.ni.c om), which provides full automation for each trapping routine. The power of the optical trap laser transmission was set to $40.7 \mathrm{~mW}$. The TIRF image was recorded from $0 \mathrm{sec}$, the trap was turned on at $1 \mathrm{sec}$, the translation stage movement of $10 \mu \mathrm{m}$ at $2 \mu \mathrm{m} \mathrm{s}^{-1}$ begins at $5 \mathrm{sec}$ and ends at $10 \mathrm{sec}$, the trap was deactivated at $11 \mathrm{sec}$ and the image recording stopped at $22 \mathrm{sec}$ (related to 11 -sec recovery periods). A $10-\mu \mathrm{m}$ distance threshold was chosen to ensure consistency between experiments in which chloroplasts were moved; in particular, $10 \mu \mathrm{m}$ was chosen as it is not so far as to generate potential side effects such as pushing the chloroplast into the tonoplast, yet is far enough to move chloroplasts a substantial distance from their original position.

\section{Micrograph quantification}

Chloroplast quantification was performed automatically using a MATLAB script. Stromules were manually counted from maximumprojection Z-stacks. The percentage of chloroplasts with stromules was calculated by dividing the number of chloroplasts with stromules by the total number of chloroplasts.

The quantification of haustorial-chloroplast-nucleus accumulation was performed manually from original confocal micrographs by looking through each and every layer of the Z-stack with only the brightfield and $P$. infestans 88069 td channels active to identify haustoria without bias. The associations of chloroplasts and/or nuclei with these marked haustoria were then counted, assessing each layer of the Z-stack as opposed to viewing a Z-projection that could lead to false positives. For Figure $1 c$ and Figure S4b, two individuals independently quantified chloroplast-haustoria associations, with discrepancies re-checked to reach a consensus quantification.

\section{Mock haustoria}

Mock haustoria were applied to each of the confocal micrographs that were used in the data sets that contributed to Figures 1a and 2 using standard IMAGEJ line tools. All channels were turned off except the brightfield and the P. infestans 88069td channel to reduce bias. To position the mock haustorium, a straight line was drawn that bisected the base (where the haustorium enters the plant cell) to the tip of each actual haustorium, extending across the vacuole until the cell border opposite was hit (as seen from the brightfield). This end position was taken to be the position at which the mock haustorium penetrated the cell. The line width was set to $3 \mu \mathrm{m}$ (based on the general observation that haustoria were $2.7 \mu \mathrm{m}$ in width). All channels were turned back on and the region around the mock haustorium and the instances of chloroplast-nucleus presence adjacent to the mock haustorium were counted; note that only the $Z$-slices containing the actual haustorium from which the mock was positioned were counted.

This method was used to keep the number of mock and actual haustoria per cell similar, for comparison, but in the following cases a mock haustorium could not be placed: if the mock haustorium position overlaps with an actual haustorium; if the cell border is out of the field of view; and if the actual haustorium has developed in the crook of a cell and is touching both cell borders. If no mock haustoria could be successfully placed, the image was not included in the pair-wise comparison.

\section{Statistical analysis}

The statistical significance of any differences observed when comparing means (stromule quantification) was assessed by Student's $t$-test when found to be normal by the Shapiro-Wilk test. If data were found to be non-normally distributed, a Wilcox statistical test was implemented in R. A pair-wise Wilcoxon test was used to compare mock versus actual haustoria accumulation (Figures $\mathrm{S} 1 \mathrm{~b}$ and S6), with samples paired by data coming from the same micrograph. Counts of haustoria-chloroplast-nucleus association were pooled together to generate an overall proportion/percentage from all micrographs, instead of treating each micrograph as a technical repeat and taking the percentage association as a single data point. This was done to avoid micrographs that contained only one haustorium, and would therefore generate many 100 and $0 \%$ values, skewing the mean estimate. The proportions of each observation were compared using a Fisher's exact test in $\mathrm{R}$ where statistical comparison was made.

\section{Chloroplast automated counting algorithm through image processing}

Image processing algorithms were used to calculate the gradient of the image to identify the boundaries of the puncta. Enclosed 
regions formed by the boundaries were algorithmically identified and counted. This procedure was performed for each individual channel: green (in chloroplast stroma) and blue (chloroplast autofluorescence). The chloroplasts (GFP channel) containing stromules were counted in a semi-automated fashion.

\section{Western blotting}

Protein extraction, purification and Western blot analysis steps were performed as described previously (Bozkurt et al., 2011). Anti-phosphorylated MAPK (Phospho-p44/42 MAPK; Cell Signaling Technology, https://www.cellsignal.co.uk) was used as the primary antibody; anti-rabbit antibody (Sigma-Aldrich) was used as secondary antibody.

\section{ACKNOWLEDGEMENTS}

We thank Dr Alex Jones (Warwick) for initiating the collaboration with IS, Dr Sebastian Schornack (SLCU) for initiating collaboration with MS, Prof. Peter Nixon (Imperial) for providing CpGFP plant seeds and Prof. Savithramma Dinesh-Kumar for providing us with the RecA-cTP HyPer construct. TOB's lab is funded by the Biotechnology and Biological Sciences Research Council (BBSRC; BB/ M002462/1). The Facility for Imaging by Light Microscopy (FILM) at Imperial College London is part supported by funding from the Wellcome Trust (grant 104931/Z/14/Z) and BBSRC (grant BB/ L015129/1).

\section{AUTHOR CONTRIBUTIONS}

Conceptualization: ZS, CD, MES, MS, IS and TOB. Data curation: ZS, CD, AT, PP, LHY, DCAG, BCB, IS and TOB. Formal analysis: $Z S, C D, A T, P P, Y L, B C B$, IS and TOB. Funding acquisition: TOB. Investigation: $Z S, C D, A T, P P, Y L, M E S, L H Y, A Y L, V K, B C B$ and TOB. Methodology: ZS, CD, AT, YL, DCAG, ADW, SWB, BCB, MS, IS and TOB. Project administration: ZS, CD and TOB. Resources: ZS, CD, AT, AYL, VK, IP, IS and TOB. Software: ZS and IP. Supervision: $Z S, C D, A T, P P, M E S, B C B$, IS and TOB. Validation: $Z S, C D$, $A T, P P, Y L, M E S, Y T, A D W, M S$ and IS. Visualization: $Z S, C D$ and TOB. Original draft preparation: $Z S, C D$ and TOB. Review and editing: ZS, CD, AT, PP, MES, LHY, DCAG, AYL, ADW, SWB, MS, IS and TOB.

\section{CONFLICT OF INTEREST}

The authors declare that they have no conflicts of interest associated with this work.

\section{DATA AVAILABILITY STATEMENT}

All data are available in the main text and the supporting information; raw data are available upon request. We are happy to provide all materials used here upon request.

\section{SUPPORTING INFORMATION}

Additional Supporting Information may be found in the online version of this article.

Video S1. 3D image of Figure 1a showing chloroplast focal accumulation at haustoria and stromules interacting with each other and other chloroplasts.

Video S2. Time-lapse series showing chloroplasts moving around a haustorium.

Video S3. 3D time-lapse series showing dynamic stromule interactions and relocation of chloroplasts towards a haustorium.

Video S4. Time-lapse series two chloroplasts navigating towards a haustorium without producing stromules.
Video S5. Time-lapse series showing chloroplasts accumulation to a haustorium.

Video S6. Time-lapse series showing collapse of haustorium associated with a chloroplast.

Video S7. Time-lapse series showing collapse of haustorium associated with a chloroplast.

Video S8. Time-lapse series showing the optical trapping of a chloroplast in Figure 1d that escapes the trap and springs back to the haustorium.

Video S9. Time-lapse series showing chloroplasts and stromules moving around a haustorium.

Video S10. 3D image of Figure S7a showing chloroplasts form long-distance stromule interactions that can bridge more than one haustorium.

Video S11. 3D image of chloroplast and stromules embracing a haustorium.

Video S12. Time-lapse series showing the optical trapping of a chloroplast in Figure 3d and the co-migration of a second chloroplast interacting via a stromule-like extension.

Figure S1. Mock haustoria positioned opposite real haustoria are less frequently adjacent to chloroplasts.

Figure S2. Increasing concentrations of LatA cause increasingly visible disruption to the normal actin filaments.

Figure S3. Oryzalin treatment does not affect chloroplast-haustoria association or stromule induction.

Figure S4. Alternative lighting regimes do not affect the association of chloroplasts with haustoria.

Figure S5. Chloroplasts of a haustoriated cell can be associated with haustoria and the nucleus in a non-mutually exclusive manner.

Figure S6. Haustoria association with chloroplasts alone, or chloroplasts and the nucleus together, occurs at a higher frequency than expected from chance.

Figure S7. Stromules often bridge multiple chloroplasts, including those associated with different haustoria.

Figure S8. PAMPs induce MAPK phosphorylation at the concentrations shown to induce stromules.

Figure S9. VIGS $B A K 1$ construct successfully reduces expression of $B A K 1$.

Figure S10. AVR3a induces cell death in the presence of R3a.

\section{REFERENCES}

Belhaj, K., Lin, B. \& Mauch, F. (2009) The chloroplast protein RPH1 plays a role in the immune response of Arabidopsis to Phytophthora brassicae. Plant Journal, 58(2), 287-298. https://doi.org/10.1111/j.1365-313X.2008. 03779.x.

Belousov, V.V., Fradkov, A.F., Lukyanov, K.A., Staroverov, D.B., Shakhbazov, K.S., Terskikh, A.V. et al. (2006) Genetically encoded fluorescent indicator for intracellular hydrogen peroxide. Nature Methods, 3(4), 281286. https://doi.org/10.1038/nmeth866.

Bozkurt, T.O., Belhaj, K., Dagdas, Y.F., Chaparro-Garcia, A., Wu, C.H., Cano, L.M. et al. (2015) Rerouting of plant late endocytic trafficking toward a pathogen interface. Traffic, 16(2), 204-226. https://doi.org/10.1111/tra.12245.

Bozkurt, T.O., Richardson, A., Dagdas, Y.F., Mongrand, S., Kamoun, S. \& Raffaele, S. (2014) The plant membrane-associated REMORIN1.3 accumulates in discrete perihaustorial domains and enhances susceptibility to phytophthora infestans. Plant Physiology, 165(3), 1005-1018. https:// doi.org/10.1104/pp.114.235804.

Bozkurt, T.O., Schornack, S., Win, J., Shindo, T., llyas, M., Oliva, R. et al. (2011) Phytophthora infestans effector AVRblb2 prevents secretion of a plant immune protease at the haustorial interface. Proceedings of the National Academy of Sciences of the United States of America, 108(51), 20832-20837. https://doi.org/10.1073/pnas.1112708109. 
Brunkard, J.O., Runkel, A.M. \& Zambryski, P.C. (2015) Chloroplasts extend stromules independently and in response to internal redox signals. Proceedings of the National Academy of Sciences of the United States of America, 112(32), 10044-10049. https://doi.org/10.1073/pnas. 1511570112.

Caplan, J.L., Kumar, A.S., Park, E., Padmanabhan, M.S., Hoban, K., Modla, S. et al. (2015) Chloroplast stromules function during innate immunity Developmental Cell, 34(1), 45-57. https://doi.org/10.1016/j.devcel.2015.05. 011.

Chaparro-Garcia, A., Schwizer, S., Sklenar, J., Yoshida, K., Petre, B., Bos, J.I.B. et al. (2015) Phytophthora infestans RXLR-WY effector AVR3a associates with dynamin-related protein 2 required for endocytosis of the plant pattern recognition receptor FLS2. PLoS One, 10(9), e0137071. https://doi.org/10.1371/journal.pone.0137071.

Chaparro-Garcia, A., Wilkinson, R.C., Gimenez-lbanez, S., Findlay, K., Coffey, M.D., Zipfel, C. et al. (2011) The receptor-like kinase serk3/bak1 is required for basal resistance against the late blight pathogen Phytophthora infestans in Nicotiana benthamiana. PLoS One, 6(1), e16608. https://doi.org/10.1371/journal.pone.0016608.

Dagdas, Y.F., Pandey, P., Tumtas, Y., Sanguankiattichai, N., Belhaj, K., Duggan, C. et al. (2018) Host autophagy machinery is diverted to the pathogen interface to mediate focal defense responses against the irish potato famine pathogen. ELife, 7, 1-15. https://doi.org/10.7554/eLife.37476.

Daniel, R. \& Guest, D. (2006) Defence responses induced by potassium phosphonate in Phytophthora palmivora-challenged Arabidopsis thaliana. Physiological and Molecular Plant Pathology, 67(3-5), 194-201. https://doi.org/10.1016/j.pmpp.2006.01.003.

de Torres Zabala, M., Littlejohn, G., Jayaraman, S., Studholme, D., Bailey, T., Lawson, T. et al. (2015) Chloroplasts play a central role in plant defence and are targeted by pathogen effectors. Nature Plants, 1(6) 15074. https://doi.org/10.1038/NPLANTS.2015.74.

Ding, X., Jimenez-Gongora, T., Krenz, B. \& Lozano-Duran, R. (2019) Chloroplast clustering around the nucleus is a general response to pathogen perception in Nicotiana benthamiana. Molecular Plant Pathology, 20(9), 1298-1306. https://doi.org/10.1111/mpp.12840.

Erickson, J.L., Adlung, N., Lampe, C., Bonas, U. \& Schattat, M.H. (2018) The Xanthomonas effector XopL uncovers the role of microtubules in stromule extension and dynamics in Nicotiana benthamiana. Plant Journal, 93(5), 856-870. https://doi.org/10.1111/tpj.13813.

Genre, A., Chabaud, M., Timmers, T., Bonfante, P. \& Barker, D.G. (2005) Arbuscular mycorrhizal fungi elicit a novel intracellular apparatus in Medicago truncatula root epidermal cells before infection. The Plant Cell, 17(12), 3489-3499. https://doi.org/10.1105/tpc.105.035410.

Gray, J.C., Hansen, M.R., Shaw, D.J., Graham, K., Dale, R., Smallman, P. et al. (2012) Plastid stromules are induced by stress treatments acting through abscisic acid. Plant Journal, 69(3), 387-398. https://doi.org/10. 1111/j.1365-313X.2011.04800.x.

Griffis, A.H.N., Groves, N.R., Zhou, X. \& Meier, I. (2014) Nuclei in motion: movement and positioning of plant nuclei in development, signaling, symbiosis, and disease. Frontiers in Plant Science, 5(APR), 1-7. https:// doi.org/10.3389/fpls.2014.00129.

Hanson, M.R. \& Hines, K.M. (2018) Stromules: probing formation and function. Plant Physiology, 176(1), 128-137. https://doi.org/10.1104/pp.17. 01287.

Heese, A., Hann, D.R., Gimenez-lbanez, S., Jones, A.M.E., He, K., Li, J. et al. (2007) The receptor-like kinase SERK3/BAK1 is a central regulator of innate immunity in plants. Proceedings of the National Academy of Sciences of the United States of America, 104(29), 12217-12222. https:// doi.org/10.1073/pnas.0705306104

Helle, S.C.J., Kanfer, G., Kolar, K., Lang, A., Michel, A.H. \& Kornmann, B. (2013). Organization and function of membrane contact sites. Biochimica et Biophysica Acta (BBA) - Molecular Cell Research, 1833(11), 2526-2541. https://doi.org/10.1016/j.bbamcr.2013.01.028

Hellens, R., Mullineaux, P. \& Klee, H. (2000) A guide to Agrobacterium binary Ti vectors. Trends in Plant Science, 5(10), 446-451. https://doi.org/10. 1016/S1360-1385(00)01740-4

Higa, T., Suetsugu, N., Kong, S.G. \& Wada, M. (2014) Actin-dependent plastid movement is required for motive force generation in directional nuclear movement in plants. Proceedings of the National Academy of Sciences of the United States of America, 111(11), 4327-4331. https://doi. org/10.1073/pnas.1317902111.
Hoitzing, H., Johnston, I.G. \& Jones, N.S. (2015) What is the function of mitochondrial networks? A theoretical assessment of hypotheses and proposal for future research. BioEssays, 37(6), 687-700. https://doi.org/ 10.1002/bies.201400188.

Jelenska, J., Yao, N., Vinatzer, B.A., Wright, C.M., Brodsky, J.L. \& Greenberg, J.T. (2007) A J domain virulence effector of pseudomonas syringae remodels host chloroplasts and suppresses defenses. Current Biology, 17(6), 499-508. https://doi.org/10.1016/j.cub.2007.02.028.

Jones, J.D.G. \& Dangl, J.L. (2006). The plant immune system. Nature, 444 (7117), 323-329. https://doi.org/10.1038/nature05286

Kadota, A., Yamada, N., Suetsugu, N., Hirose, M., Saito, C., Shoda, K. et al. (2009) Short actin-based mechanism for light-directed chloroplast movement in Arabidopsis. Proceedings of the National Academy of Sciences of the United States of America, 106(31), 13106-13111. https://doi.org/10. 1073/pnas.0906250106.

Kamoun, S., Van West, P., Vleeshouwers, V.G.A.A., De Groot, K.E. \& Govers, F. (1998) Resistance of Nicotiana benthamiana to Phytophthora infestans is mediated by the recognition of the elicitor protein INF1. The Plant Cell, 10(9), 1413-1425. https://doi.org/10.1105/tpc.10.9.1413.

Ketelaar, T., Meijer, H.J.G., Spiekerman, M., Weide, R. \& Govers, F. (2012) Effects of latrunculin $B$ on the actin cytoskeleton and hyphal growth in Phytophthora infestans. Fungal Genetics and Biology, 49(12), 1014-1022. https://doi.org/10.1016/j.fgb.2012.09.008.

Kobayashi, I. \& Hakuno, H. (2003) Actin-related defense mechanism to reject penetration attempt by a non-pathogen is maintained in tobacco BY-2 cells. Planta, 217(2), 340-345. https://doi.org/10.1007/s00425-003-1042-3.

Koh, S., André, A., Edwards, H., Ehrhardt, D. \& Somerville, S. (2005) Arabidopsis thaliana subcellular responses to compatible Erysiphe cichoracearum infections. Plant Journal, 44(3), 516-529. https://doi.org/10.1111/j. 1365-313X.2005.02545.x.

Kumar, A.S., Park, E., Nedo, A., Alqarni, A., Ren, L., Hoban, K. et al. (2018) Stromule extension along microtubules coordinated with actin-mediated anchoring guides perinuclear chloroplast movement during innate immunity. ELife, 7, 1-33. https://doi.org/10.7554/eLife.23625.

Kwok, E.Y. \& Hanson, M.R. (2003) Microfilaments and microtubules control the morphology and movement of non-green plastids and stromules in Nicotiana tabacum. Plant Journal, 35(1), 16-26. https://doi.org/10.1046/j. 1365-313X.2003.01777.x.

Kwon, C., Neu, C., Pajonk, S., Yun, H.S., Lipka, U., Humphry, M. et al. (2008) Co-option of a default secretory pathway for plant immune responses. Nature, 451(7180), 835-840. https://doi.org/10.1038/nature06545.

Liu, L. \& Li, J. (2019). Communications between the endoplasmic reticulum and other organelles during abiotic stress response in plants. Frontiers in Plant Science, 10, 749. https://doi.org/10.3389/fpls.2019.00749.

Lukan, T., Županič, A., Mahkovec Povalej, T., Brunkard, J.O., Juteršek, M. Baebler, S. et al. (2021) Chloroplast redox state changes indicate cell-tocell signalling during the hypersensitive response. BioRxiv.

Natesan, S.K.A., Sullivan, J.A. \& Gray, J.C. (2009) Myosin XI is required for actin-associated movement of plastid stromules. Molecular Plant, 2(6), 1262-1272. https://doi.org/10.1093/mp/ssp078.

Oikawa, K., Yamasato, A., Kong, S.G., Kasahara, M., Nakai, M., Takahashi F. et al. (2008) Chloroplast outer envelope protein Chup1 is essential for chloroplast anchorage to the plasma membrane and chloroplast movement. Plant Physiology, 148(2), 829-842. https://doi.org/10.1104/pp.108. 123075

Opalski, K.S., Schultheiss, H., Kogel, K.H. \& Hückelhoven, R. (2005) The receptor-like MLO protein and the RAC/ROP family G-protein RACB modulate actin reorganization in barley attacked by the biotrophic powdery mildew fungus Blumeria graminis f.sp. hordei. Plant Journal, 41(2), 291 303. https://doi.org/10.1111/j.1365-313X.2004.02292.x.

Padmanabhan, M.S. \& Dinesh-Kumar, S.P. (2010) All hands on deck-the role of chloroplasts, endoplasmic reticulum, and the nucleus in driving plant innate immunity. Molecular Plant-Microbe Interactions, 23(11), 13681380. https://doi.org/10.1094/MPMI-05-10-0113.

Pecrix, Y., Buendia, L., Penouilh-Suzette, C., Maréchaux, M., Legrand, L. Bouchez, O. et al. (2019) Sunflower resistance to multiple downy mildew pathotypes revealed by recognition of conserved effectors of the oomycete Plasmopara halstedii. Plant Journal, 97(4), 730-748. https://doi.org/ 10.1111/tpj.14157.

Petre, B., Lorrain, C., Saunders, D.G.O., Win, J., Sklenar, J., Duplessis, S. et al. (2016) Rust fungal effectors mimic host transit peptides to 
translocate into chloroplasts. Cellular Microbiology, 18(4), 453-465. https://doi.org/10.1111/cmi.12530.

Rocchetti, A., Hawes, C. \& Kriechbaumer, V. (2014) Fluorescent labelling of the actin cytoskeleton in plants using a cameloid antibody. Plant Methods, 10(1), 12. https://doi.org/10.1186/1746-4811-10-12.

Schattat, M., Barton, K., Baudisch, B., Klösgen, R.B. \& Mathur, J. (2011) Plastid stromule branching coincides with contiguous endoplasmic retic ulum dynamics. Plant Physiology, 155(4), 1667-1677. https://doi.org/10. 1104/pp.110.170480.

Schattat, M.H., Barton, K.A. \& Mathur, J. (2015) The myth of interconnected plastids and related phenomena. Protoplasma, 252(1), 359-371. https:// doi.org/10.1007/s00709-014-0666-4.

Scheler, B., Schnepf, V., Galgenmüller, C., Ranf, S. \& Hückelhoven, R (2016) Barley disease susceptibility factor RACB acts in epidermal cel polarity and positioning of the nucleus. Journal of Experimental Botany, 67(11), 3263-3275. https://doi.org/10.1093/jxb/erw141.

Schmelzer, E. (2002) Cell polarization, a crucial process in fungal defence. Trends in Plant Science, 7(9), 411-415. https://doi.org/10.1016/S1360-1385 (02)02307-5.

Schneider, C.A., Rasband, W.S. \& Eliceiri, K.W. (2012). NIH Image to ImageJ: 25 years of image analysis. Nature Methods, 9(7), 671-675. https://doi. org/10.1038/nmeth.2089

Silva, B.S.C., DiGiovanni, L., Kumar, R., Carmichael, R.E., Kim, P.K. \& Schrader M. (2020) Maintaining social contacts: The physiological relevance of organelle interactions. Biochimica et Biophysica Acta (BBA) - Molecular Cell Research, 1867(11), 118800. https://doi.org/10.1016/j.bbamcr.2020.118800

Song, J., Win, J., Tian, M., Schornack, S., Kaschani, F., llyas, M. et al. (2009). Apoplastic effectors secreted by two unrelated eukaryotic plant pathogens target the tomato defense protease Rcr3. Proceedings of the National Academy of Sciences, 106(5), 1654-1659. https://doi.org/10. 1073/pnas.0809201106

Sparkes, I., White, R.R., Coles, B., Botchway, S.W. \& Ward, A. (2018). Using optical tweezers combined with total internal reflection microscopy to study interactions between the ER and Golgi in plant cells. Methods in Molecular Biology, 1691, 167-178.

Stegemann, S., Keuthe, M., Greiner, S. \& Bock, R. (2012) Horizontal transfer of chloroplast genomes between plant species. Proceedings of the National Academy of Sciences of the United States of America, 109(7), 2434-2438. https://doi.org/10.1073/pnas.1114076109.

Su, J., Yang, L., Zhu, Q., Wu, H., He, Y., Liu, Y. et al. (2018) Active photosynthetic inhibition mediated by MPK3/MPK6 is critical to effector-triggered immunity. PLoS Biology, 16(5), 1-29. https://doi.org/10.1371/journal.pbio. 2004122.
Suetsugu, N., Higa, T., Gotoh, E. \& Wada, M. (2016) Light-induced movements of chloroplasts and nuclei are regulated in both $\mathrm{cp}$ actin-filament-dependent and -independent manners in arabidopsis thaliana. PLoS One, 11(6), e0157429. https://doi.org/10.1371/journal.pone. 0157429.

Suetsugu, N. \& Wada, M. (2016) Evolution of the Cp-Actin-based motility system of chloroplasts in green plants. Frontiers in Plant Science, 7, 561. https://doi.org/10.3389/fpls.2016.00561.

Tang, C., Deng, L., Chang, D., Chen, S., Wang, X. \& Kang, Z. (2016) TaADF3, an Actin-Depolymerizing factor, negatively modulates wheat resistance against puccinia striiformis. Frontiers in Plant Science, 6, 1-14. https:// doi.org/10.3389/fpls.2015.01214.

Van Damme, M., Zeilmaker, T., Elberse, J., Andel, A., De Sain-van Der Velden, M. \& Van Den Ackerveken, G. (2009) Downy mildew resistance in arabidopsis by mutation of Homoserine Kinase. The Plant Cell, 21(7) 2179-2189. https://doi.org/10.1105/tpc.109.066811.

Van West, P., De Jong, A.J., Judelson, H.S., Emons, A.M.C. \& Govers, F. (1998) The ipiO gene of phytophthora infestans is highly expressed in invading hyphae during infection. Fungal Genetics and Biology, 23(2), 126-138. https://doi.org/10.1006/fgbi.1998.1036

Wada, M. \& Kong, S.G. (2018) Actin-mediated movement of chloroplasts. Journal of Cell Science, 131(2). https://doi.org/10.1242/jcs.210 310

Wang, S., Boevink, P.C., Welsh, L., Zhang, R., Whisson, S.C. \& Birch, P.R.J. (2017) Delivery of cytoplasmic and apoplastic effectors from Phytophthora infestans haustoria by distinct secretion pathways. New Phytologist, 216(1), 205-215. https://doi.org/10.1111/nph.14696.

Wang, Y., Li, X., Fan, B., Zhu, C. \& Chen, Z. (2021) Regulation and function of defense-related callose deposition in plants. International Journal of Molecular Sciences, 22(5), 1-15. https://doi.org/10.3390/ ijms22052393

Whisson, S.C., Boevink, P.C., Moleleki, L., Avrova, A.O., Morales, J.G., Gilroy, E.M. et al. (2007) A translocation signal for delivery of oomycete effector proteins into host plant cells. Nature, 450(7166), 115-118. https:// doi.org/10.1038/nature06203.

Whisson, S.C., Boevink, P.C., Wang, S. \& Birch, P.R. (2016) The cell biology of late blight disease. Current Opinion in Microbiology, 34, 127-135. https://doi.org/10.1016/j.mib.2016.09.002

Wu, C.H., Abd-El-Haliem, A., Bozkurt, T.O., Belhaj, K., Terauchi, R., Vossen, J.H. et al. (2017) NLR network mediates immunity to diverse plant pathogens. Proceedings of the National Academy of Sciences of the United States of America, 114(30), 8113-8118. https://doi.org/10.1073/pnas. 1702041114. 